The Astrophysical Journal, 655:416-433, 2007 January 20

(C) 2007. The American Astronomical Society. All rights reserved. Printed in U.S.A.

\title{
FEATURES OF THE ACOUSTIC MECHANISM OF CORE-COLLAPSE SUPERNOVA EXPLOSIONS
}

\author{
A. Burrows, ${ }^{1}$ E. Livne, ${ }^{2}$ L. Dessart,${ }^{1}$ C. D. Ott ${ }^{3}$ and J. Murphy ${ }^{1}$ \\ Received 2006 July 20; accepted 2006 September 27
}

\begin{abstract}
In the context of 2D, axisymmetric, multigroup, radiation/hydrodynamic simulations of core-collapse supernovae over the full $180^{\circ}$ domain, we present an exploration of the progenitor dependence of the acoustic mechanism of explosion. All progenitor models we have tested with our Newtonian code explode. However, some of the cores left behind in our simulations, particularly for the more massive progenitors, have baryon masses that are larger than the canonical $\sim 1.5 M_{\odot}$ of well-measured pulsars. We investigate the roles of the standing accretion shock instability (SASI), the excitation of core $g$-modes, the generation of core acoustic power, the ejection of matter with $r$-process potential, the windlike character of the explosion, and the fundamental anisotropy of the blasts. We find that the breaking of spherical symmetry is central to the supernova phenomenon, the delays to explosion can be long, and the blasts, when top-bottom asymmetric, are self-collimating. We see indications that the initial explosion energies are larger for the more massive progenitors and smaller for the less massive progenitors and that the neutrino contribution to the explosion energy may be an increasing function of progenitor mass. However, the explosion energy is still accumulating by the end of our simulations and has not converged to final values. The degree of explosion asymmetry we obtain is completely consistent with that inferred from the polarization measurements of Type Ic supernovae. Furthermore, we calculate for the first time the magnitude and sign of the net impulse on the core due to anisotropic neutrino emission and suggest that hydrodynamic and neutrino recoils in the context of our asymmetric explosions afford a natural mechanism for observed pulsar proper motions.
\end{abstract}

Subject headings: hydrodynamics — neutrinos — radiation mechanisms: general — stars: oscillations (including pulsations) — supernovae: general

\section{INTRODUCTION}

One of the outstanding problems in astrophysics is the mechanism of core-collapse supernova explosions. This pedigreed puzzle has resisted more than 40 years of theoretical speculation and numerical exploration. It has intrigued many unaware of the numerous feedbacks that mute the consequences of most alterations in microphysical processes. It has tricked specialists with a legendary list of false starts and blind alleys. It has taunted computational astrophysicists with both its imagined and real complexities. The potential roles as factors in explosion of neutrinos, the nuclear equation of state (EOS), exotic physics, general relativity, dimensionality, instabilities, magnetic fields, and rotation all continue to be topical.

The neutrino heating mechanism, in which a stalled bounce shock is reenergized by neutrino energy deposition after a slight delay, perhaps aided by overturning instabilities in this "gain region," has been the working hypothesis of the community for the last 20 years (Bethe \& Wilson 1985; Buras et al. 2006a, 2006b; Liebendörfer et al. 2001). Past calculations in support of this mechanism, or variations on its theme, include those by Wilson \& Mayle (1988, 1993), Mayle \& Wilson (1988), Herant et al. (1994), Burrows et al. (1995), Janka \& Müller (1996), and Fryer \& Warren $(2002,2004)$.

Nevertheless, recent calculations employing careful neutrino physics and numerics suggest that the neutrino mechanism, when it succeeds, may at best be marginal. Kitaura et al. (2006) follow in

\footnotetext{
1 Department of Astronomy and Steward Observatory, University of Arizona, Tucson, AZ; burrows@as.arizona.edu, luc@as.arizona.edu,jmurphy@as.arizona .edu.

2 Racah Institute of Physics, Hebrew University, Jerusalem, Israel; eli@frodo .fiz.huji.ac.il.

3 Max-Planck-Institut für Gravitationsphysik, Albert-Einstein-Institut, Golm/ Potsdam, Germany; cott@aei.mpg.de.
}

spherical symmetry the compact $1.38 M_{\odot} \mathrm{O}-\mathrm{Ne}-\mathrm{Mg}$ core of the $8.8 M_{\odot}$ model of Nomoto \& Hashimoto (1988), with a very tenuous outer envelope, and obtain a delayed neutrino-driven explosion. However, the explosion energy is only $\gtrsim 10^{50}$ ergs and the major driver of the explosion is the neutrino-driven wind (Burrows 1987; Burrows \& Goshy 1993; Janka et al. 2005a, 2005b). Buras et al. (2006b) witness the onset of the SASI-aided, ${ }^{4}$ neutrinodriven $\ell=1$ explosion of the $11.2 M_{\odot}$ progenitor of Woosley et al. (2002, hereafter WHW02). Curiously, they also infer a very weak explosion energy, this time near $10^{49} \mathrm{ergs}$, not correcting for neutrino driving subsequent to the early termination of their calculation or for the binding energy of the outer envelope. Buras et al. (2006b) focus on the importance of calculating over the full $180^{\circ}$ two-dimensional (2D) domain so as not to suppress the $\ell=1$ mode of the SASI, since they do not obtain even a weak explosion when constraining the computational domain to $90^{\circ}$. They also imply that performing the calculations in 3D might make the explosion robust.

However, perhaps the most interesting conclusion of the Buras et al. (2006b) paper is that of all of the progenitors they study (including $11.2,13,15,20$, and $25 M_{\odot}$ models), only the 11.2 $M_{\odot}$ star, with its steep outer density gradient, small iron core $\left(\sim 1.26 M_{\odot}\right)$, and close-in $\mathrm{Si} / \mathrm{O}$ density shelf, approaches the $\tau_{\text {adv }} \sim \tau_{H}$ condition, promoted by Thompson et al. (2005) as the litmus test for the neutrino-driven explosion mechanism. ${ }^{5}$ Furthermore, Buras et al. (2006b) find that only the $11.2 M_{\odot}$ progenitor amply satisfies the mantle overturn and perturbation

\footnotetext{
4 SASI: standing accretion shock instability; see Blondin et al. (2003), Blondin \& Mezzacappa (2006), Foglizzo \& Tagger (2000), Foglizzo (2001, 2002), Foglizzo et al. (2005, 2007), Buras et al. (2006a, 2006b), and Burrows et al. (2006).

5 Here $\tau_{\text {adv }}$ is the timescale for shocked matter to traverse the gain region and $\tau_{H}$ is the neutrino heating timescale. When $\tau_{\text {adv }}$ is long and/or $\tau_{H}$ is short, neutrino heating can explode the shocked mantle.
} 
growth condition (Foglizzo et al. 2006) they evoke to analyze the potential for the SASI in the first $\sim 200 \mathrm{~ms}$ after bounce.

From the work of Kitaura et al. (2006), Buras et al. (2006b), and Janka et al. (2005a, 2005b) one might conclude that the neutrino mechanism requires compact cores and tenuous outer envelopes, but that for such progenitors a solely neutrino-driven explosion is perforce weak. The small accretion rates that may be necessary after bounce for the neutrino mechanism to succeed ensure that when it does succeed there is little mass to absorb the driving neutrino luminosity, resulting in the weak explosion. The neutrino-driven mechanism alone, and on its own, may not be able to yield a robust, $\sim 10^{51}$ erg explosion.

The large mass accretion rates experienced by more massive cores after bounce create more massive postshock absorbing/gain regions and higher neutrino luminosities, but those same accretion rates may well tamp and suppress the neutrino-driven explosion. However, for the more massive progenitors that do not now seem to explode by the neutrino mechanism, it may be that better neutrino transfer, coupled with and aided by an $\ell=1$ SASI mode calculated over the full $180^{\circ}$ domain, will follow the path of the 11.2 $M_{\odot}$ progenitor as simulated by Buras et al. (2006b) and result in explosions. Three-dimensional simulations with sophisticated neutrino numerics and physics have not been performed and may well reveal qualitative, or large quantitative, differences with the results in two dimensions. The neutrino mechanism may yet prove to be more muscular and universal. However, it is also possible that the typical supernova of the typical massive-star progenitor does not explode by the neutrino mechanism, or solely by the neutrino mechanism, and that another, nonmagnetic ${ }^{6}$ mechanism may be at work.

Recently, Burrows et al. (2006) have proposed an acoustic mechanism for exploding core-collapse supernovae. In it, the progressive growth of the SASI and of the entropy and Mach number of the accreted shocked matter long after the outer shock has stalled results in anisotropic accretion onto the inner core that over time excites core $g$-modes. ${ }^{7}$ Predominantly $\ell=1$ in character, these core eigenmodes achieve large amplitudes and dampen by the radiation of sound. Multiple sound pulses emanate from the core with periods of 2-4 ms and steepen into shock waves. The resulting acoustic power deposits energy and momentum aspherically into the outer shocked mantle and explodes the supernova, but on timescales of many hundreds of milliseconds to seconds. ${ }^{8}$ The blast is fundamentally aspherical, favoring one side. During the early explosion, the other side continues to experience accretion, which maintains the core oscillation and the generation of sound until the entire mantle has exploded. Thus, the breaking of spherical symmetry and the excitation and maintenance of aspherical $g$-modes allow simultaneous accretion and explosion. As long as it is needed to ensure success, the core acts like a transducer for the conversion of accretion gravitational power into outwardly propagating acoustic power. Curiously,

\footnotetext{
6 Although we suspect that strong magnetic fields require very rapid rotation that may not be available in the generic core-collapse supernova context (Ott et al. 2006a), magnetic jets have been suggested as potential power sources (Akiyama et al. 2003; Kotake et al. 2006; Moiseenko et al. 2006; Obergaulinger et al. 2006). Perhaps rapid rotation could facilitate an MHD scenario for the rare hypernovae and long/soft gamma-ray bursts (GRBs), or for accretion-induced collapse (Dessart et al. 2006b).

The SASI itself is insufficient to explode the supernova. Contrary to Blondin et al. (2003), when proper account is taken of the neutrino losses, the nuclear EOS, and the inner boundary, the total transverse turbulent kinetic energy in the shocked zone does not grow (Burrows et al. 2006; Buras et al. 2006a, 2006b).

${ }^{8}$ If another mechanism (such as the neutrino mechanism) were to explode the envelope significantly earlier, the inner core oscillations might not be excited to importance and the core acoustic model might be aborted.
}

the recoil due to the resulting anisotropic mass loss pushes the accretion streams that are exciting the core oscillation into a configuration that is even more favorable for the excitation of $\ell=1$ core oscillations ( $\S 6$; Burrows et al. 2006). In this way, the core $g$-modes seem self-excited. This is what we observe in our simulations, but such an intriguing phenomenon remains to be verified. Perhaps, even if the initial explosion is neutrino-driven and it too is very asymmetrical due to the $\ell=1$ SASI mode, the recoil due to the anisotropic neutrino-driven mass loss can, by the same mechanism, excite core $g$-mode oscillations. Hence, even a neutrino-triggered explosion might excite core oscillations, which would radiate acoustic power and boost the explosion energy. Such a "hybrid" mechanism for supernova explosions is a particularly intriguing possibility but has yet to be adequately explored.

One may further speculate that there is not one core-collapse supernova mechanism, but several. The lowest mass massive star progenitors (Kitaura et al. 2006; Buras et al. 2006b) and accretion-induced collapse (Dessart et al. 2006b) might explode early and subenergetically by the neutrino-driven wind mechanism, while the generic progenitor might explode by the acoustic mechanism (aided by neutrino heating). However, when better neutrino transport than we have employed is included, we may find that the neutrino/acoustic hybrid mechanism obtains throughout most of the progenitor mass range, particularly if the explosion commences after the vigorous phase of the $\ell=1$ SASI begins, with the relative contributions of neutrino heating and acoustic power varying as a function of progenitor mass. Finally, the subset with rapidly rotating cores, or cores that collapse into black holes before explosion, might rely on MHD processes to explode the mantle, and these might be associated with hypernovae and/or GRBs. All in all, the outcome will depend on the progenitor's inner density and rotation profiles.

Our calculations support the notion that all nonrotating progenitors that do not explode by the early neutrino mechanism experience the SASI and later excite core pulsations that generate acoustic power that aids or enables explosion. ${ }^{9}$ In this paper we present the results of preliminary investigations into the progenitor dependence of the core oscillation/acoustic mechanism. In $\S 2$ we compare the density profiles of representative nonrotating progenitor models of Woosley \& Weaver (1995, hereafter WW95), WHW02, and Nomoto \& Hashimoto (1988) and discuss the resulting mass accretion and proto-neutron star mass accumulation histories. These profiles determine the outcome of collapse. In $\S 3$ we discuss the overall hydrodynamic behavior of some of these models, focusing on the evolution of the shock position, the dependence of the SASI frequency on progenitor profile, and the core pulsation energy. In $\S 4$ we discuss the entropies achieved, the degree of radiation domination, and the possible consequences for the $r$-process. We then go on in $\S 5$ to examine the aspherical windlike character of the explosion and its consequences and the anisotropy of the neutrino emissions and the resulting recoils. This leads us to suggest a natural model for pulsar kicks. Finally, in $\S 6$ we summarize and discuss our conclusions concerning the core oscillation/acoustic mechanism of core-collapse supernova explosions.

For these simulations, we have used the 2D multigroup, multineutrino-species, flux-limited diffusion (MGFLD) variant of the code VULCAN/2D (Livne et al. 2004; Walder et al. 2005; Ott et al. 2006a, 2006b; Dessart et al. 2006a, 2006b; Burrows et al. 2006) and describe many of its numerical features in the Appendix. This is currently the only extant $2 \mathrm{D}$, multigroup code that

\footnotetext{
${ }^{9}$ Unless the core first collapses to a black hole.
} 
allows core translational motion by introducing a Cartesian-like grid in the inner core and, hence, that is capable of investigating the core oscillation/acoustic mechanism. VULCAN/2D is also the only extant supernova code to perform 2D (not "ray by ray") multigroup transport. Due to the finite difference character of 2D codes that employ spherical coordinates all the way to the center, to the singularity in those coordinates at that center, and to the reflecting boundary condition frequently imposed at this center, spherical coordinate codes are likely to inhibit core translational motions artificially and, hence, to inhibit the $\ell=1 \mathrm{~g}$-modes that are central to the mechanism we have identified. Be that as it may, there are many caveats to our study the reader should keep in mind: (1) our calculations are Newtonian and not general relativistic; (2) as stated above, we employ an approximate multigroup transport algorithm in the neutrino sector; (3) numerical errors are bound to have accumulated due to the need to calculate for $\sim 1,000,000$ time steps for each progenitor; and (4) the initial seed perturbations are unknown (and unknowable?). Furthermore, the flow is fundamentally chaotic and a precise mapping between initial configuration and final outcome is not possible. This multidimensional radiation/hydrodynamical problem is quintessentially meteorological in character. Nevertheless, along with the work of Burrows et al. (2006), these are the first calculations to explore the novel core oscillation/acoustic mechanism and to venture into the late-time behavior of multidimensional core collapse with multidimensional core motions and multidimensional/ multigroup transport.

\section{PROGENITOR DENSITY PROFILES AND MASS ACCUMULATION RATES}

The basic evolutionary phases through which a core proceeds in the context of the core oscillation/acoustic supernova mechanism have been described in Burrows et al. (2006), to which the reader is referred for details. These are summarized in $\S \S 1$ and 6. Burrows et al. (2006) explored the results for the $11 M_{\odot}$ model of WW95 alone. Since that paper, we have calculated more models, including the $25 M_{\odot}$ model of WW95 and the rotating m15b6 model of Heger et al. (2005) (Ott et al. 2006a, 2006b), as well as the $11.2,13,15,20$, and $25 M_{\odot}$ progenitor models of WHW02 and the 13 and $15 M_{\odot}$ models of Nomoto \& Hashimoto (1988). All models explode, modulo any fallback at very, very late times not yet accessible to supernova codes. This set of models constitutes the most extensive and detailed radiation/hydrodynamic study of the shock instability (SASI) and of the multidimensional core motions undertaken to date.

For nonrotating models, the most important determinant of the outcome of collapse is the density profile in the inner thousands of kilometers of the massive star progenitor. The structure of this "Chandrasekhar" core, with surrounding inner envelope, is determined by the burning history to the point of instability. This history reflects the various core- and shell-burning stages and is a function in nature of zero-age main-sequence (ZAMS) mass, mass loss, and metallicity. However, different theoretical groups performing calculations of the evolution of massive stars and using different approaches to semiconvection, overshoot, convection, and mass loss still do not end with the same configurations. Figure 1 provides some density profiles for progenitor models from WHW02, Nomoto \& Hashimoto (1988), and WW95 at a point just after collapse ensues. The first thing to note is that there is a spread in structures and that the Chandrasekhar core is not the same for all progenitors, but varies in structure and mass. The corresponding $Y_{e}$ and entropy profiles vary similarly. Secondly, as a comparison of the two sets of 13 and $15 M_{\odot}$ models shown in Figure 1 makes clear, the structure for a given progenitor mass has

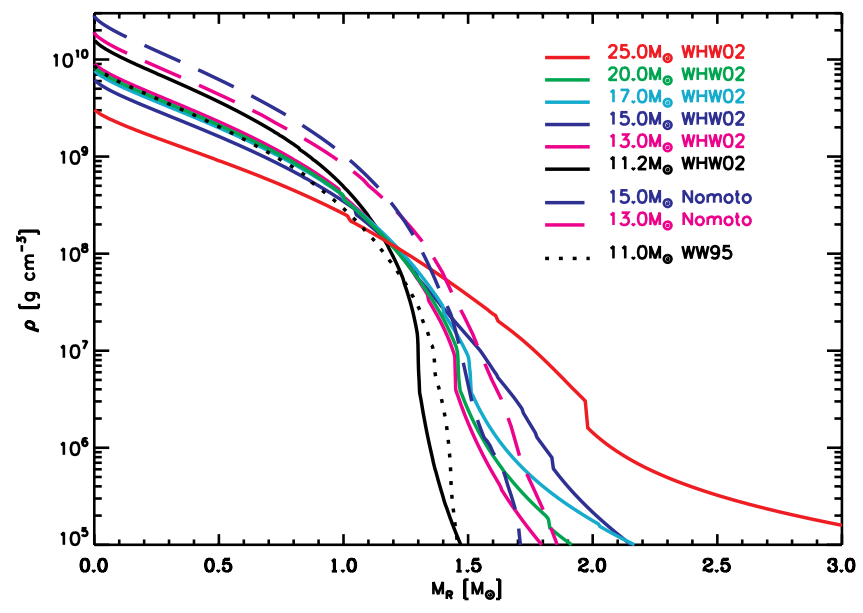

FIG. 1.-Profiles of the mass density (in units of $\mathrm{g} \mathrm{cm}^{-3}$ ) vs. Lagrangean mass (in $M_{\odot}$ ) of representative massive star progenitor cores of WHW02 (11.2 $M_{\odot}$ : black; $13 M_{\odot}:$ magenta; $15 M_{\odot}:$ blue; $17 M_{\odot}$ : turquoise; $20 M_{\odot}:$ green; $25 M_{\odot}:$ red $)$, Nomoto \& Hashimoto (1988; $13 M_{\odot}$ : magenta; $15 M_{\odot}$ : blue), and WW95 (11 $M_{\odot}$ : dotted line). See text for a discussion on the import of these profiles.

not converged theoretically. Different groups arrive at different profiles for the same ZAMS mass. Thirdly, the density profiles are not necessarily monotonic with ZAMS progenitor mass: the $15 M_{\odot}$ model of WHW02 has a shallower profile than that of their $20 M_{\odot}$ model, and the $15 M_{\odot}$ model of Nomoto \& Hashimoto (1988) has a steeper profile than that of their $13 M_{\odot}$ model. Finally, the older $11 M_{\odot}$ model of WW95 and the more recent one of WHW02 at 11.2 $M_{\odot}$, while both being steep, are not equally steep in the same regions. The 11.2 $M_{\odot}$ model has lower densities between interior masses from 1.2 to $1.45 M_{\odot}$, while the $11 M_{\odot}$ model of WW95 has lower densities exterior to that mass (not shown). The upshot is that the outcome of collapse and the character of whatever explosion is ignited are not likely to be the same. In particular, the 11.2 $M_{\odot}$ model of WHW02 boasts the thinnest mantle of their whole model set, and this is consistent with the explanation given in $\S 1$ for why Buras et al. (2006b) obtained an SASIand neutrino-aided explosion, albeit weak, but no such explosion for the more massive cores with shallower and thicker density profiles. The even steeper profile of the ONeMg model of Nomoto \& Hashimoto (1988) (not shown in Fig. 1) explains the results of Kitaura et al. (2006), and the near vacuum of the outer envelopes used in the accretion-induced collapse simulations of Dessart et al. (2006b) explains why they saw weak neutrino-aided explosions.

The structures depicted in Figure 1 translate directly into mass accretion rates $(\dot{M})$ through the stalled shock. Because the inner shocked region and the core are out of sonic contact with this mantle, $\dot{M}$ and its evolution after bounce are functions of this structure alone. Hence, for diagnosing and "predicting" the outcome of collapse, the postbounce behavior of $\dot{M}$ for a given progenitor is useful and probably determinative. Figure 2 portrays the evolution of the mass accretion rate for representative progenitor models evolved using the 2D MGFLD variant of VULCAN/ 2D. The wide range of curves reflects the range of profiles plotted in Figure 1. At $0.5 \mathrm{~s}$ after bounce, $\dot{M}$ varies from 0.06 to $0.5 M_{\odot} \mathrm{s}^{-1}$, while at $1.0 \mathrm{~s}$ it varies from 0.02 to $0.3 M_{\odot} \mathrm{s}^{-1}$, an order-of-magnitude span at both epochs. A glance at the behavior of $\dot{M}$ for the 11.2 $M_{\odot}$ model used in Buras et al. (2006b) shows its steep drop at early times and the corresponding lower accretion tamp. Such a precipitous drop is not in evidence for the other, more massive WHW02 progenitors portrayed in Figure 2. 


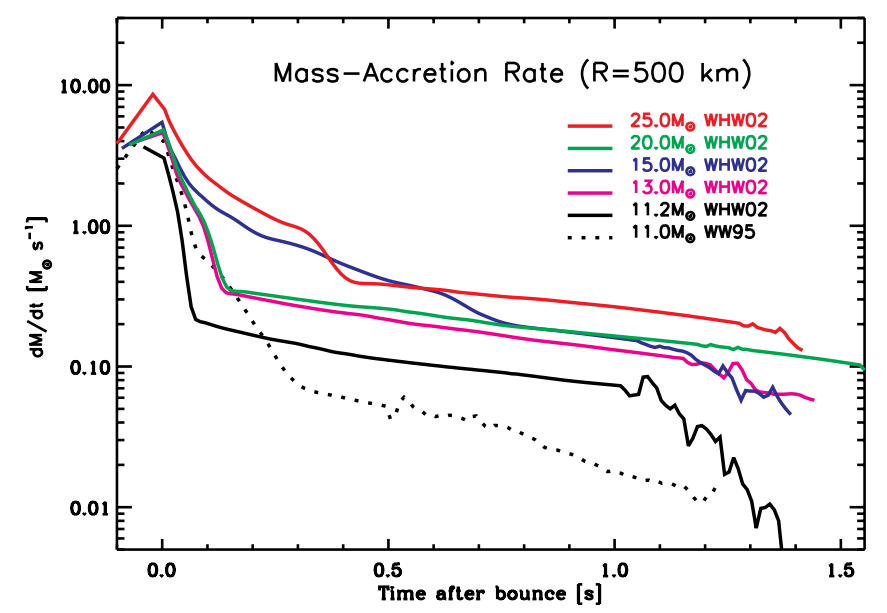

FIG. 2.-Pre- and postbounce time evolution of the accretion rate through a radius of $500 \mathrm{~km}$ (in $M_{\odot} \mathrm{s}^{-1}$ ), for various massive star progenitors. Only the infalling matter is included. The color coding and line styles are as in Fig. 1.

The plummeting of $\dot{M}$ at later times for the $11 M_{\odot}$ model of WW95 used in Burrows et al. (2006) is tied to the earlier onset of the sound-powered explosion they witness, earlier than we generally find in this paper, employing as we do here a fuller range of progenitor models with shallower density profiles. The drops in $\dot{M}$ seen at the latest times shown in Figure 2 are consequences of the late-onset acoustic-driven explosions we find, with the $11.2 M_{\odot}$ model exploding earliest. Probably because our MGFLD neutrino transfer can still be improved, we do not reproduce the weak neutrino-driven explosion seen by Buras et al. (2006b) for the 11.2 $M_{\odot}$ model. However, determining the precise reasons for the difference will entail a direct comparison of the details of both codes, something that will be subtle. As we show in Dessart et al. (2006a), the luminosities and matter profiles are generally similar for the two codes. The Buras et al. (2006b) calculations were done using a GR substitute; ours were Newtonian. The Buras et al. (2006b) calculation does a remap between their Eulerian (PPM) hydro code and comoving frame (Lagrangian) transport. Ours does Lagrangian hydro and then remaps to an Eulerian grid. Buras et al. (2006b) calculate spherical transport along radial rays, using for each ray the same Eddington factor for an average sphere. We do flux-limited diffusion, but in full 2D. Buras et al. (2006b) do not include the lateral (angular) fluxes in the transport update of the radiation fields; we do. We each use a different number of energy groups, placed at different neutrino energies. One difference is our neglect of the velocity-dependent terms in the transport equation. Buras et al. (2006b) calculate the radiation field in the comoving frame and conclude that their inclusion decreases the net gain. We do the calculations in the laboratory frame. Hubeny \& Burrows (2006) have included the velocity terms in such a laboratory frame formalism and find that the effect increases the net gain by $\sim 10 \%$. The sign depends on the frame in which you calculate the radiation quantities. Hence, if we were to include the velocity terms, it would lead to an increase in the neutrino heating. However, whether that would make a qualitative difference in our $2 \mathrm{D} 11.2 M_{\odot}$ simulation remains to be seen.

Figure 3 shows the evolution of the baryon mass accumulated in the proto-neutron star for a subset of progenitors simulated with VULCAN/2D. When corrected for the binding energy shed during deleptonization and neutrino cooling (Burrows \& Lattimer 1986), these masses can be used to provide the gravitational masses of the neutron stars (or black holes) that remain.

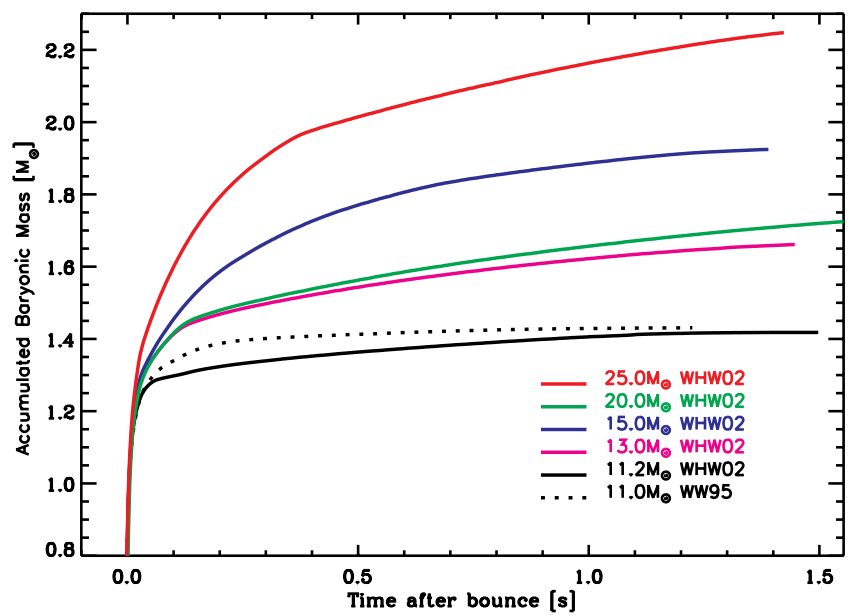

FIG. 3.-Time evolution after core bounce of the baryonic mass interior to $100 \mathrm{~km}$ of the nascent proto-neutron star for representative massive star progenitors. See text for discussion.

This plot also enables one to estimate the residual baryon mass if the explosion were to occur earlier, since the $\dot{M}$ evolution is fixed by the progenitor structure (Fig. 1). One can insert in the plot a vertical line at a given time and read off the baryon mass remaining if the explosion of a given progenitor core were to occur at that time, or one can insert a horizontal line at a given mass to determine the time a given core must explode to leave that baryon mass behind. For instance, Figure 3 indicates that for the $20 M_{\odot}$ progenitor of WHW02 to leave a neutron star with a baryon mass less than $1.5 M_{\odot}$ (equivalent to a gravitational mass of $\sim 1.35 M_{\odot}$ ), it must explode before $0.3 \mathrm{~s}$ after bounce. Similarly, for the $25 M_{\odot}$ model of WHW02 to leave such a neutron star, it must explode within the first 50-100 ms of bounce. However, the 11 and $11.2 M_{\odot}$ models need not explode before $1.5 \mathrm{~s}$ to leave behind an object with a baryon mass of $1.4 M_{\odot}$ (roughly equivalent to a gravitational mass of $\sim 1.28 M_{\odot}$ ). In addition, Figure 3 can be used to determine the maximum time to the explosion of a given progenitor, and for a given nuclear EOS, if a neutron star, and not a black hole, is to result (ignoring any fallback). Once the mechanism for explosion and the actual progenitor structures have been clearly determined, Figure 3 can also help inform any discussion concerning the progenitor mass at which the bifurcation between neutron star and black hole final products occurs.

Figure 3 indicates that, for the more massive models shown, leaving behind neutron stars with baryon masses less than 1.6 $M_{\odot}$ would require earlier explosions than we currently obtain. Such "early" explosions may require relativistic calculations, better neutrino transport, better numerics, different progenitor models, or $3 \mathrm{D}$ effects. However, in this paper we focus on the general, qualitative effects that emerge from our investigations and do not claim at this preliminary stage, given the remaining compromises in our computational approach (see the Appendix), to have arrived at final numbers.

\section{HYDRODYNAMIC COMPARISONS OF DIFFERENT PROGENITORS}

Figure 4 depicts the evolution with time after bounce of the radial positions along the poles (in the positive and negative directions) of the outer shock for three representative progenitor model simulations with VULCAN/2D. The delay to explosion for all models is $\sim 1 \mathrm{~s}$, with the stars with the steepest initial density gradients (Fig. 1) and the lowest $\dot{M}$ values (Fig. 2) exploding 


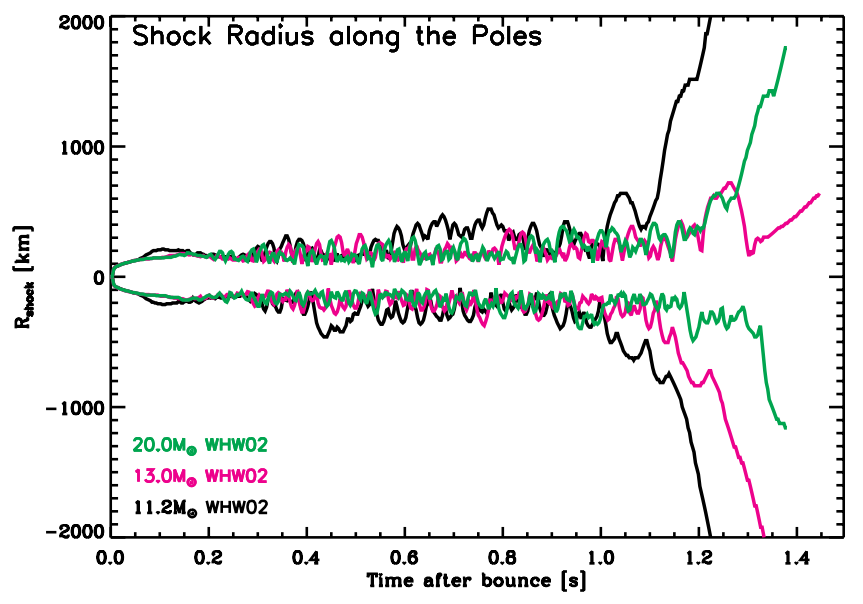

FIG. 4.-Time evolution of the outer shock radius (in $\mathrm{km}$ ) along the poles for the $11.2 M_{\odot}$ (black), $13 M_{\odot}$ (magenta), and $20 M_{\odot}$ (green) models of WHW02. The radii extend from 2000 to $-2000 \mathrm{~km}$.

earliest. Although the $11.2 M_{\odot}$ model of WHW02 does not explode within the first $\sim 100 \mathrm{~ms}$ of bounce, its shock radius is consistently larger and experiences larger excursions before explosion than the other models shown in Figure 4. The excursions during the SASI phase range from $\sim 150$ to $\sim 500 \mathrm{~km}$ and can be quite dramatic, and the explosions, when they eventually occur, are
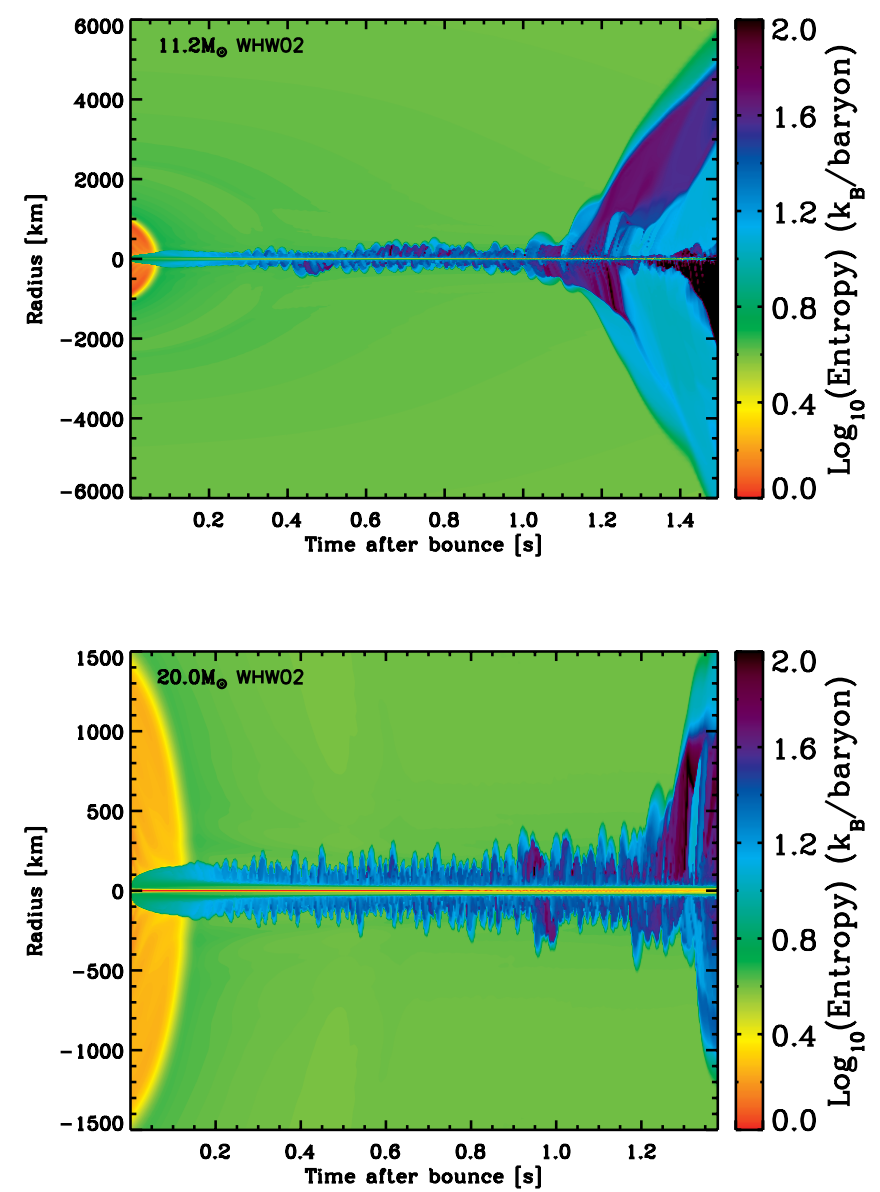

unmistakable. After the explosion ensues, it takes only $\sim 100 \mathrm{~ms}$ for the shock to reach $\sim 1000 \mathrm{~km}$. By the end of all of the simulations performed for this study, the explosion radius has reached $\sim 6500 \mathrm{~km}$ or more along multiple directions.

However, as Figure 4 demonstrates and Figure 5, which depicts a color map of the \pm polar entropy profile for four representative models, confirms, the blasts are top-bottom asymmetric. The SASI and the core oscillation represent symmetry breaking, and the direction of explosion depends on the chaotic evolution of the flow and the timing of the explosion. We see explosions that are very unipolar (e.g., our $25 M_{\odot}$ run) and more top-bottom symmetric (e.g., our 11.2 $M_{\odot}$ run). One cannot predict ahead of time in which direction the core will explode, nor the degree of anisotropy. However, one can expect that the distribution of the top-bottom asymmetries and the character of these asymmetries can eventually be determined statistically. Figure 6 portrays snapshots of the explosion debris a few hundred milliseconds after explosion of the 11.2 and $20 M_{\odot}$ models and highlights the different degrees of early blast asymmetry we can expect. We have seen top-bottom asymmetries larger than that for the $20 M_{\odot}$ model, but none less than that of the 11.2 $M_{\odot}$ model. Note that we are not concluding anything about the likely progenitor dependence of the explosion asymmetry. On the contrary, we are merely documenting the diversity we see in this set of numerical realizations.

As Figure 2 demonstrates, the evolution of the mass accretion rate is different for the different progenitors. Among other things,
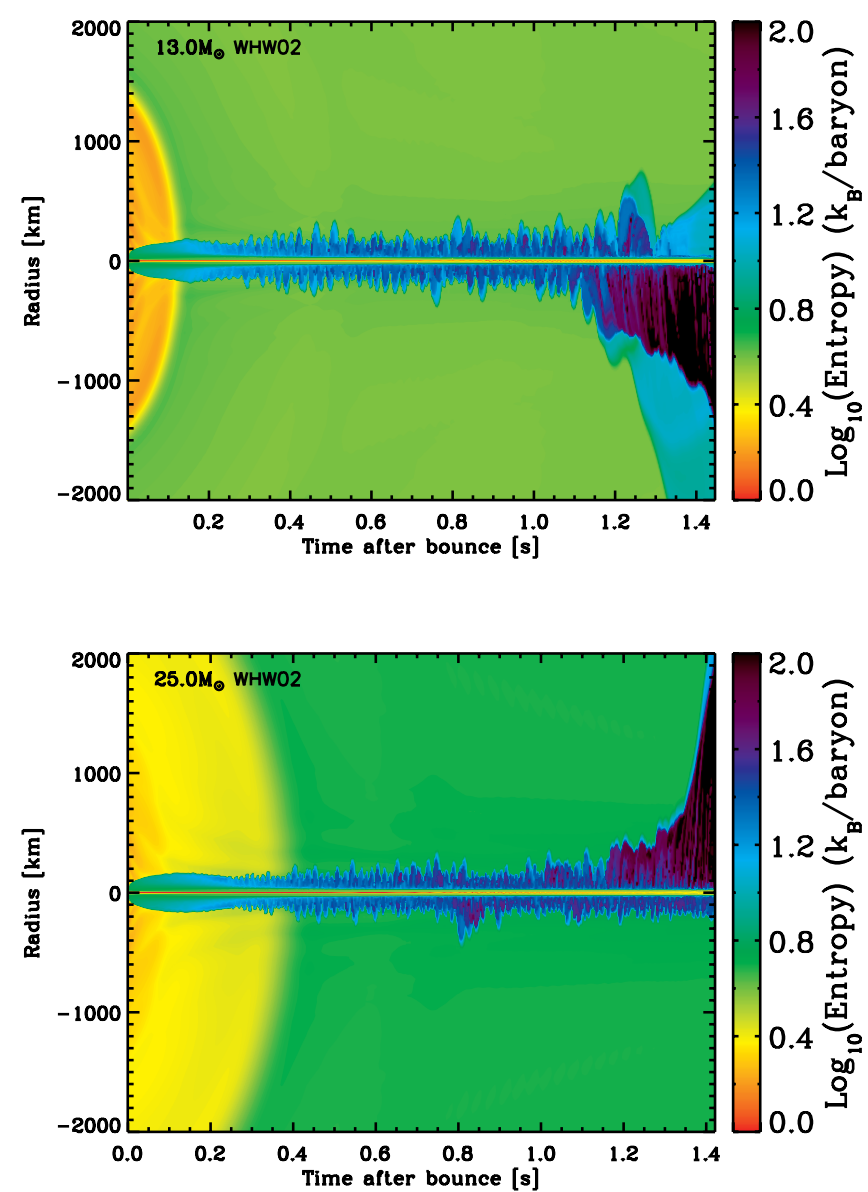

FIG. 5.-Time evolution of the entropy profiles along the poles of the $11.2 M_{\odot}$ (top left), $13 M_{\odot}$ (top right), $20 M_{\odot}$ (bottom left), and $25 M_{\odot}$ (bottom right) models of WHW02. The positions of the shocks are clearly indicated by the abrupt transition from the green color (low entropy) of the infalling material. Color bars indicating the values of the logarithm of the entropy (per baryon per Boltzmann constant) are provided on the right sides of each panel and go from red (entropy $\sim 1$ ) to purple (entropy $\geq 100$ per baryon per Boltzmann's constant). 

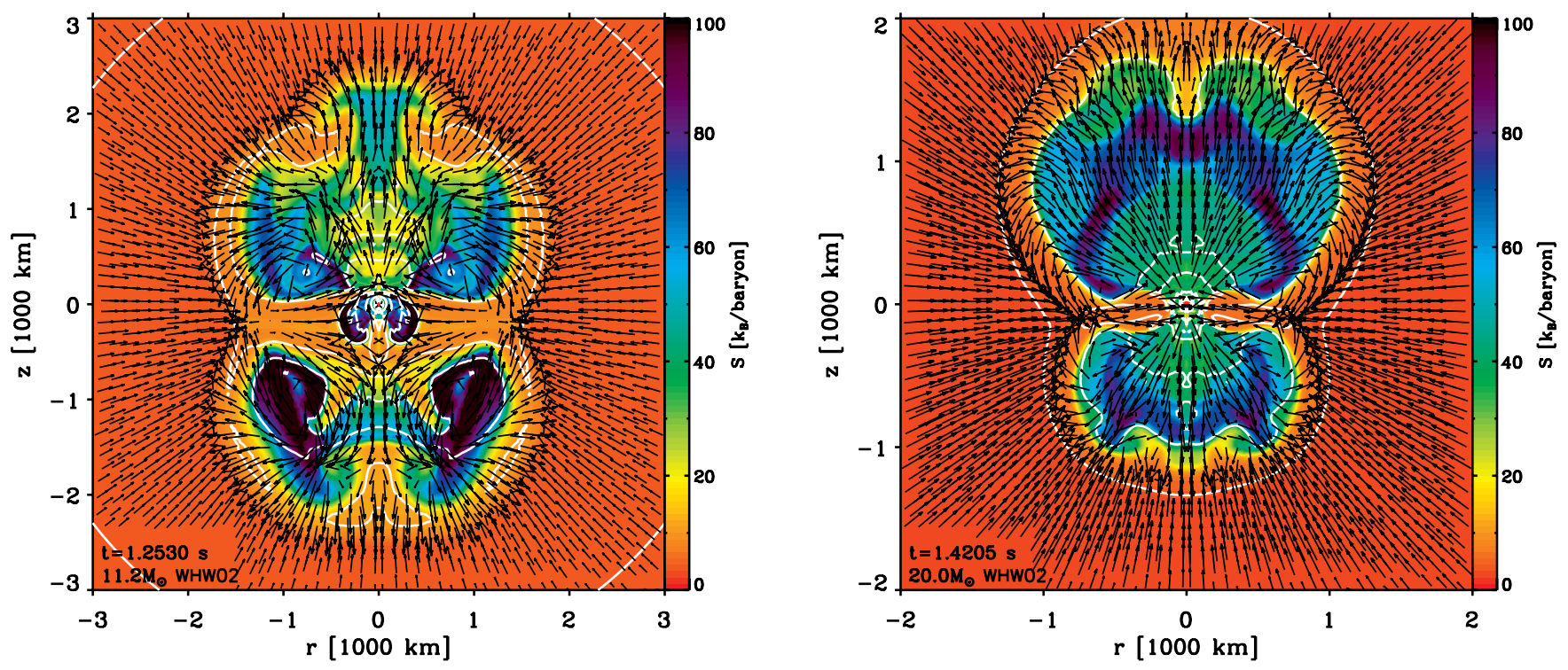

Fig. 6.-Entropy color map for the $11.2 M_{\odot}$ (left) and $20 M_{\odot}$ (right) models of WHW02. Times after bounce are indicated in the lower left corner of each panel. The vector length has been saturated at a value of $10,000 \mathrm{~km} \mathrm{~s}^{-1}$, relevant only for the infalling matter exterior to the shock.

this translates into accretion rams that are very different from model to model. Table 1 demonstrates another consequence: the SASI shock frequencies vary by more than a factor of 2.5 from progenitor to progenitor and there is a one-to-one relationship between the $\dot{M}$ and the SASI frequency. We have Fourier analyzed the shock position, and it is the dominant frequencies that are listed in Table 1, along with the average accretion rates and average shock radii during the nonlinear SASI phase before explosion. Oscillation frequencies from $\sim 30$ to $\sim 80 \mathrm{~Hz}$ are seen, and these are inversely proportional to the average radius of the stalled shock (in Table 1, from $\sim 120$ to $\sim 250 \mathrm{~km}$ ). As might have been expected, the monotonicity is with $\dot{M}$ and the shallowness of the density profile, and not the progenitor ZAMS mass. The oscillation periods implicit in Table 1 are approximately the sound-travel times across the shocked regions. Note that this is not a statement about the growth timescale of the SASI, which is very different and is not the sound-travel time (Foglizzo et al. 2006). For the smaller average shock radii that we obtain when the $\dot{M}$ values are larger, this translates into the higher oscillation frequencies for those models. This is in contrast to the similarity we see in the core $g$-mode frequencies for the various progenitors: at a given epoch this frequency ranges only modestly from

TABLE 1

SASi Frequency versus Accretion Rate and Shock Radius

\begin{tabular}{|c|c|c|c|}
\hline $\begin{array}{l}\text { WHW02 Model Mass } \\
\qquad\left(M_{\odot}\right)\end{array}$ & $\begin{array}{l}\text { Frequency } \\
\qquad(\mathrm{Hz})\end{array}$ & $\begin{array}{c}\dot{M} \\
\left(M_{\odot} \mathrm{s}^{-1}\right)\end{array}$ & $\begin{array}{c}\left\langle R_{\text {shock }}\right\rangle \\
(\mathrm{km})\end{array}$ \\
\hline 11.2 & 32 & 0.08 & 250 \\
\hline $13 \ldots$ & 47 & 0.25 & 175 \\
\hline 15 & 73 & 0.7 & 130 \\
\hline 20 & 63 & 0.3 & 155 \\
\hline $25 \ldots$ & 80 & 0.8 & 120 \\
\hline
\end{tabular}

NoTES.- $\left\langle R_{\text {shock }}\right\rangle$ is the average shock radius after the SASI becomes nonlinear, but before explosion. The SASI frequency given is for the dominant shock oscillation component during this same time interval. $\dot{M}$ is near the average accretion rate onto the proto-neutron star through a radius of $500 \mathrm{~km}$ during this same phase. model to model and, for our Newtonian calculations, the $\ell=1$ mode sticks within $\sim 30 \%$ of $\sim 300 \mathrm{~Hz}$.

As we show in Table 1, the average shock radius during the SASI phase is smaller for those progenitors with the highest postbounce mass accretion rates. The $25 M_{\odot}$ model of WHW02 is an example of a massive star progenitor with such a high rate. As demonstrated in Ott et al. (2006b), this model manifests not only $\ell=1$ core oscillations, but significant $\ell=2$ core oscillations as well. The latter are responsible for the strong gravitational radiation signature of this published model. Such strong $\ell=2$ core oscillations are more easily excited if the outer SASI shock oscillations have a strong $\ell=2$ component as well. Foglizzo et al. (2007) have recently performed an analytic stability and growth rate analysis of the SASI and find that those models with the smallest ratio between the shock radius and the inner core radius should experience stronger $\ell=2$ SASI growth. The more detailed $2 \mathrm{D}$ radiation/hydrodynamic simulations reported in this paper and in Ott et al. (2006b) for the $25 M_{\odot}$ model, with its more compact shock configuration, tend to bear out these findings. Progenitors with larger $\dot{M}$ values result in smaller shock/core radius contrasts, higher SASI frequencies, and larger growth rates for the $\ell=2$ modes of both the SASI and the core oscillation. The strong $\ell=2$ core mode can result in prodigious gravitational radiation signatures (Ott et al. 2006b) of the associated supernovae and of black hole formation, which itself may be the result of large $\dot{M}$ values.

In the left panel of Figure 7, the evolution of the net neutrino energy deposition in the gain region versus time after bounce is portrayed for five representative progenitor models from WHW02. There is a strong dependence of this power on $\dot{M}$. However, these numbers are relevant only after explosion commences and infall transitions into outflow. Before that, the net neutrino energy deposition for a given Lagrangean mass element changes sign as the settling mass element encounters the inner cooling region just exterior to the neutrinospheres. Hence, it is when these powers start to decrease due to the reduction of the neutrino luminosities caused by the decrease in $\dot{M}$ on explosion that neutrino heating can contribute to the explosion energies, and it does so in a transient fashion. As Figure 7 shows, the net effect of 

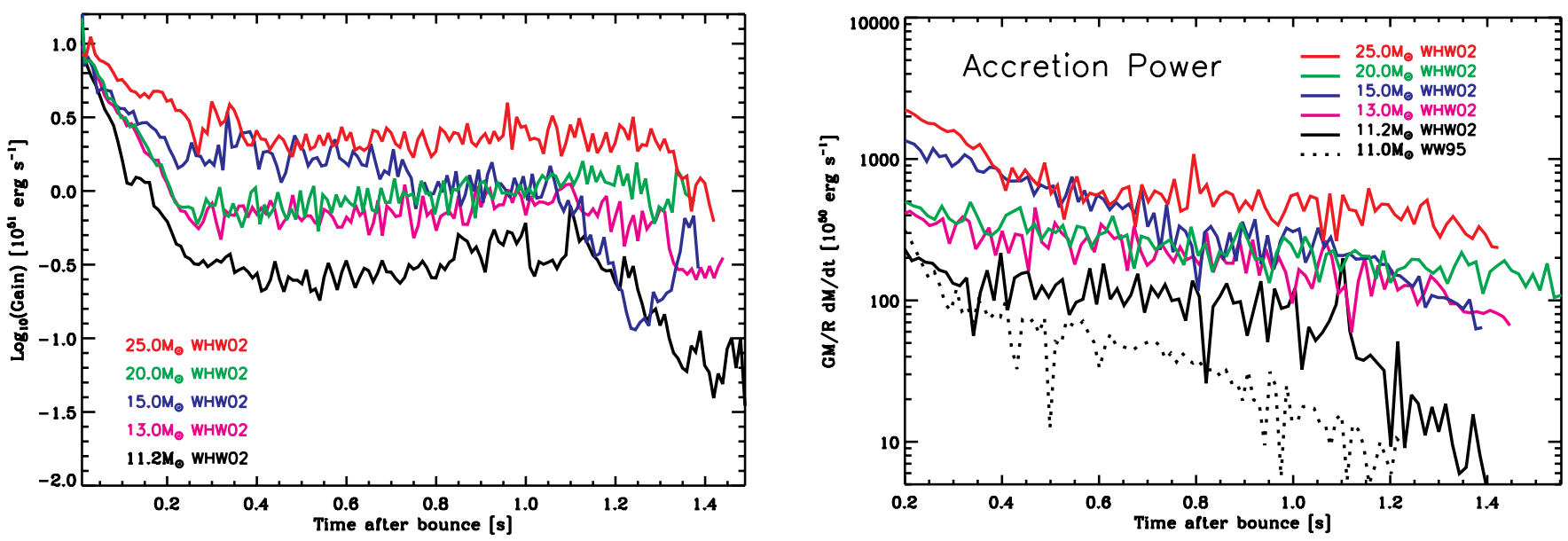

Fig. 7.-Left: Time evolution after bounce of the integrated net energy deposition due to neutrino absorption (in units of $10^{51} \mathrm{ergs} \mathrm{s}^{-1}$ ) in the gain region for representative WHW02 models $\left(11.2 M_{\odot}:\right.$ black; $13 M_{\odot}$ : magenta; $15 M_{\odot}:$ blue; $20 M_{\odot}:$ green; $25 M_{\odot}:$ red $)$. Right: Same as the left panel, but for the accretion power, defined as $\dot{M} G M / R$, where $G$ is the gravitational constant, $M$ is the mass interior to the spherical radius $R=30 \mathrm{~km}$, and $\dot{M}$ is the infall mass accretion rate at $30 \mathrm{~km}$. Also included is the $11 M_{\odot}$ model (dotted line) of WW95 calculated in Burrows et al. (2006).

neutrino heating from the onset of explosion, which itself in these calculations is due predominantly to acoustic power, is an increasing function of $\dot{M}$ and, approximately, of progenitor mass. The largest effect of neutrino heating in this model set is for the $25 M_{\odot}$ model of WHW02 and amounts to an integrated value of $\sim 2 \times 10^{50} \mathrm{ergs}$ by the end of the simulation near $1.4 \mathrm{~s}$ after bounce. At this time, the explosion is still being driven at a steady rate by acoustic power from its massive core.

In the right panel of Figure 7 we provide the corresponding evolution of the total gravitational accretion power $(M G M / R)$ for the same set of representative models, along with the values for the $11 M_{\odot}$ model studied in Burrows et al. (2006). The accretion power ranges by almost 2 orders of magnitude, directly reflecting the range in mass accretion rates (Fig. 2). Most of this power is radiated to infinity as neutrinos, without heating; only a small fraction is converted into the mechanical energy of core oscillations, most of which damps by the emission of sound. Due to the chaotic and anisotropic nature of the turbulent flow interior to the shock, it has been difficult to get an analytic handle on the efficiency of conversion of accretion power into core pulsation energy and acoustic power. However, even the small efficiency we find is enough to ignite and power explosion, after some delay. Neutrino damping of core oscillation seems to be a small effect (automatically included in our calculations), with a characteristic timescale of 5-30 s (see Ferrari et al. 2003; Miralles et al. 2004). Artificial damping due to low resolution and truncation errors seems to have a characteristic timescale longer than $3 \mathrm{~s}$. However, its magnitude is difficult to gauge, given the expense of the simulations, and must be a subject for future studies. Most of the higher resolution studies (both spatial and spectral) we have performed suggest that greater resolution leads to slightly more vigorous core oscillations and SASI, particularly when we increase the number of energy groups. Note that in the models described in this paper, the grid transitions from spherical to Cartesian at $\sim 30 \mathrm{~km}$, where the radial spacing is still a respectable $\sim 800 \mathrm{~m}$. Such resolution provides reasonable sampling ( $\sim 5$ zones per decade in density) of the steep density profile that arises in this region at late times ( $\sim 1 \mathrm{~s}$ after bounce).

Figure 8 depicts the energy in the $g$-mode oscillations of the inner core versus time after bounce for a few representative progenitor models. There seem to be two classes. The first, represented by the $11.2 M_{\odot}$ and old $11 M_{\odot}$ models with low mass accretion rates, achieves pulsation energies (kinetic plus potential) of only $\sim 10^{50} \mathrm{ergs}$. The second class is represented by the $20 M_{\odot}$ model in Figure 8, for which the core achieves pulsation energies near $\sim 10^{51}$ ergs. For such models, the turbulence of the SASI and the compactness of the shock are much greater (Table 1), and the mass accretion rates are much larger. After explosion commences, the inner cores reach quasi-steady states in which the fraction of the gravitational accretion energy channeled into mechanical energy roughly balances the acoustic losses. This happens at an acoustic power of very approximately $0.5 \times 10^{51} \mathrm{ergs} \mathrm{s}^{-1}$. The available accretion energy subsides with explosion (Fig. 7) and so the energy stored in the $g$-mode within $100-200 \mathrm{~ms}$ of the onset of explosion may be a measure of the total energy available to be pumped into the supernova "nebula" acoustically. At a loss

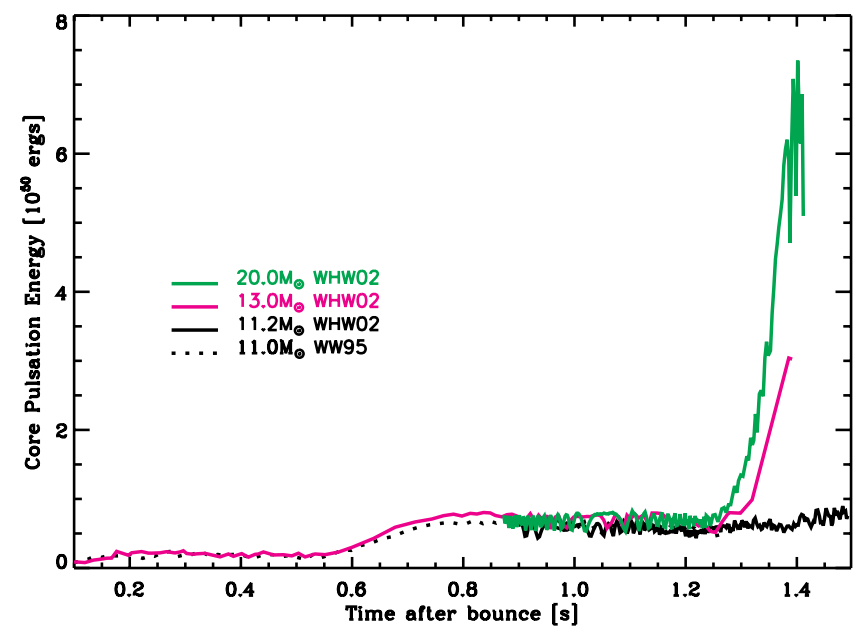

Fig. 8. - Time evolution after $0.1 \mathrm{~s}$ after bounce of the total pulsation energy (kinetic plus internal plus gravitational, in units of $10^{50} \mathrm{ergs}$ ) of the inner cores for simulations for a representative subset of progenitor models. Time is given in seconds after bounce. Note that this is not the total energy of the explosion at a given time. However, since nonsonic damping processes seem weak, the core oscillation will discharge sonically into the outer expanding "nebula" and this oscillation energy will eventually be available to the explosion. Since our calculations halted before this phase was well underway, but after the onset of explosion, we do not yet have a good estimate of the final explosion energy for these simulations. Curiously, progenitors with the largest $\dot{M}$ values seem to have the largest total pulsation energies. See text for a discussion. 

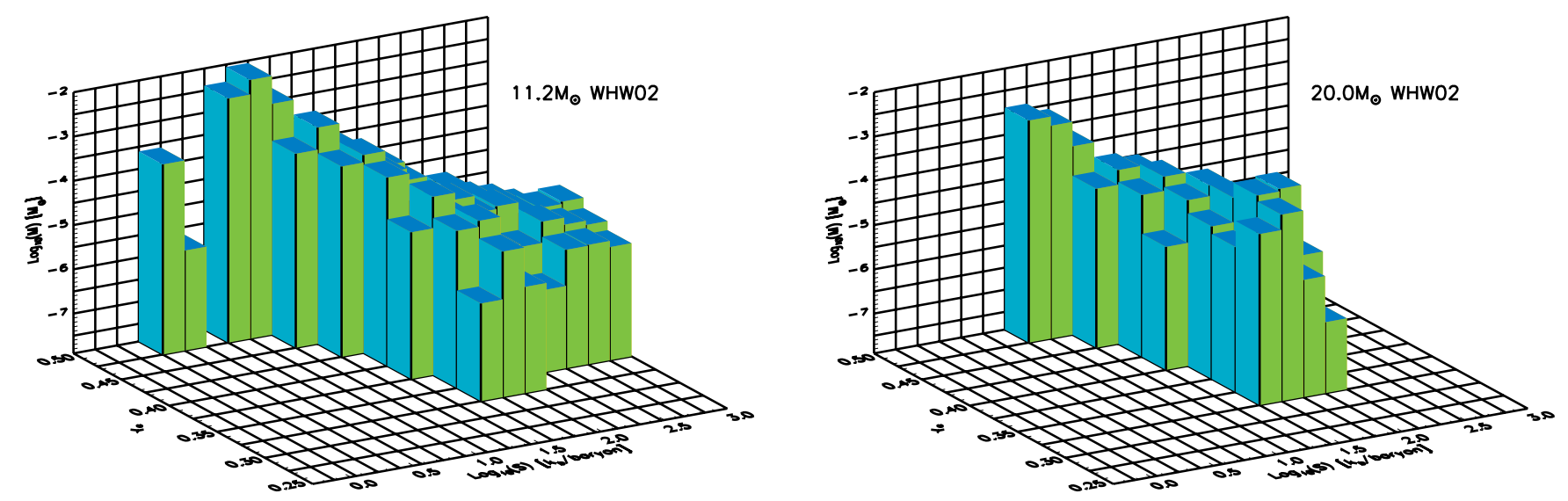

FIG. 9.- - Histograms for the $11.2 M_{\odot}(l e f t)$ and $20 M_{\odot}(r i g h t)$ models of WHW02 of the distribution of mass vs. entropy and electron fraction $\left(Y_{e}\right)$ in the explosion ejecta. The ejecta are defined as those parcels of matter having a positive total energy at $1500 \mathrm{~ms}$ after core bounce. The heights of the columns are the actual masses (actually, logarithm of the mass, in $M_{\odot}$ ). For the $11.2 M_{\odot}$ model, the total mass ejected at the end of the simulation, i.e., at $1.49 \mathrm{~s}$, is $0.0191 M_{\odot}$, while the total mass above an entropy of $100 k_{\mathrm{B}}$ baryon $^{-1}$ is $2.15 \times 10^{-4} M_{\odot}$ and above $300 k_{\mathrm{B}}$ baryon $^{-1}$ is $1.25 \times 10^{-4} M_{\odot}$. For the $20.0 M_{\odot}$ model, the total mass ejected at the end of the simulation, i.e., at $1.4 \mathrm{~s}$, is $0.004 M_{\odot}$, while the total mass above an entropy of $100 k_{\mathrm{B}}$ baryon ${ }^{-1}$ is $1.1 \times 10^{-5} M_{\odot}$ and above $300 k_{\mathrm{B}}$ baryon ${ }^{-1}$ is $0.0 M_{\odot}$.

rate of $\sim 0.5 \times 10^{51} \mathrm{ergs} \mathrm{s}^{-1}$, the low accretion rate progenitors would achieve explosion energies of a few times $10^{50} \mathrm{ergs}$ within hundreds of milliseconds and the high accretion rate progenitors would achieve explosion energies of $\sim 10^{51}$ within seconds, the time it would take their larger core oscillations to discharge acoustically. ${ }^{10}$ General relativity will increase the core frequencies and, hence, the core acoustic power, with the result that the explosion might occur earlier and, perhaps, more energetically. The dependence on the nuclear EOS is more subtle and has yet to be studied.

Whether the bifurcation into two classes is abrupt, or whether there is in reality a continuum from lower to higher core pulsation energies, remains to be seen. However, progenitors with larger mass accretion rates seem to achieve larger core pulsation energies, with the suggestion that they can explode more energetically. As the left panel of Figure 7 also suggests, the neutrino contribution to the explosion energy is expected to be larger for progenitors with higher $\dot{M}$ values at explosion, or for progenitors that for some reason explode earlier (all else being equal). Unfortunately, due to difficulties at the outer computational boundary, which must handle simultaneous infall and explosion, and/or convergence problems in the neutrino-matter coupling in the inner core material residing in the transition region from Cartesian to spherical gridding when it becomes very violently pulsational and the mass density gradients steepen precipitously, we are not yet able to evolve our models beyond $\sim 1.5 \mathrm{~s}$ after bounce. The code crashes. Hence, we do not quote total explosion energies. However, the acoustic power being pumped into the explosion $\left(\sim 10^{50}\right.$ $\left.10^{51} \mathrm{ergs} \mathrm{s}^{-1}\right)$ and the core oscillation energy ultimately available to the supernova by acoustic discharge $\left(\sim 10^{50}-10^{51}\right.$ ergs $)$ give us zeroth-order estimates of the systematics and values of the final explosion energies. From our results, the initial supernova explosion energy seems to be an increasing function of progenitor mass, when correction is made for the slight nonmonotonicities noted in Figure 1. Whether this conclusion survives will be contingent on future detailed investigations, using a variety of techniques and codes. We note that Hamuy (2003) has inferred from

\footnotetext{
10 The old $11 M_{\odot}$ model of WW95 studied in Burrows et al. (2006) exploded earlier because its inhibiting mass accretion rate was very small and because the outer boundary radius was put at too small a value $(3400 \mathrm{~km})$. The new models all have larger outer radii of $\sim 6500 \mathrm{~km}$. For the $11 M_{\odot}$ model of WW95, Burrows et al. (2006) found that the acoustic pumping lasted $\sim 400 \mathrm{~ms}$.
}

observations of supernova explosions that explosion energies might in fact span a wide range of values.

\section{ENTROPY AND ELECTRON FRACTION OF THE EJECTA}

The explosions we see involve ejecta with distributions of entropies and electron fractions $\left(Y_{e}\right)$. If the ejecta entropies achieve values in the hundreds, it has been shown that $r$-process nucleosynthesis becomes more viable (Woosley et al. 1994). We have assembled histograms of the amount of mass in the escaping fraction in the various entropy and $Y_{e}$ bins. However, since the explosions have not run to completion (despite the fact that the blasts have reached $6500 \mathrm{~km}$ and the simulations have been performed to $\sim 1.5 \mathrm{~s}$ ), we do not have final histograms for any of our simulations. Nevertheless, the numerical data we do have are intriguing and we present them in Figure 9 for the 11.2 and $20 M_{\odot}$ runs. The heights give the logarithm of the total mass in the $Y_{e}$ and entropy [actually $\log$ (entropy)] bins and are not differentials. For the 11.2 and $20 M_{\odot}$ runs, we find that the total masses ejected above entropies of 100 per baryon per Boltzmann's constant are $2.15 \times 10^{-4}$ and $1.1 \times 10^{-5} M_{\odot}$, respectively, while the total masses ejected above entropies of 300 per baryon per Boltzmann's constant are $1.25 \times 10^{-4}$ and $0.0 M_{\odot}$, respectively. The total masses ejected at any entropy are 0.0191 and $0.0041 M_{\odot}$ for the 11.2 and $20 M_{\odot}$ models, respectively. For core-collapse supernovae to be the site of the $r$-process, each must eject on average $10^{-4}$ to $10^{-5} M_{\odot}$ of $r$-process elements (Woosley \& Hoffman 1992; Woosley et al. 1994; Hoffman et al. 1996; Thompson et al. 2001). The $r$-process yield in a parcel of matter varies with entropy, $Y_{e}$, and expansion time but can be around $10 \%$ by mass for the highest entropies and the "long" expansion times (hundreds of milliseconds) we find. Most of the rest of these inner ejecta will emerge as $\alpha$-particles. The iron peak would be produced as the shock encounters and traverses the oxygen shell on timescales typically longer than those of these simulations. Note that an upper bound of 0.5 to the ejecta $Y_{e}$ was inadvertently imposed on these runs. Since $Y_{e}$ was not allowed to exceed 0.5, the potential effects of $\nu_{e}$ and $\bar{\nu}_{e}$ absorption for $Y_{e}$ values above 0.5 and/or in enabling the $\nu-p$ and $r p$-processes (Fröhlich et al. 2005, 2006a, 2006b; Pruet et al. 2005, 2006) were not properly incorporated. Nevertheless, the purpose of Figure 9 is to demonstrate that high entropies are achieved, and this conclusion is not effected by the 
" $\leq 0.5$ " constraint. Furthermore, our necessary use of an MGFLD algorithm, instead of full Boltzmann transport, for these multidimensional runs should in itself and in any case produce less reliable values for the ejecta $Y_{e}$ values.

The histogram in the left panel of Figure 9, depicting the results for the 11.2 $M_{\odot}$ model with a significant amount of ejecta above entropies of 300 , suggests that the $r$-process yield of that model is in the middle of the desired range. We have yet to postprocess our ejecta with detailed nucleosynthesis codes, and so our results are at best preliminary. However, ours are the first consistent supernova calculations that both explode and eject matter with true $r$-process potential.

The large entropies achieved in the acoustic/core oscillation mechanism are in part a consequence of the late explosion in lower density matter and of the compound effects of multiple shocks originating from the multiple sound pulses. However, neutrino heating of the matter made thinner by acoustic driving is a factor as well. Had the supernova explosion, actually a wind, been driven exclusively by neutrinos, they would have been responsible for the density profile as well. The entropies, densities, temperatures, and $Y_{e}$ values of this wind would all have been determined by the driving neutrino luminosity and would have been inadequate to achieve the high entropies necessary for $r$-process conditions (Thompson et al. 2001). However, since both acoustic and neutrino driving are simultaneously operative, the neutrinos can deposit energy in material already made thinner by the acoustic effects, resulting in higher entropies than can be achieved by neutrinos alone. Analysis of the contributions of these different agents to entropization is made next to impossible by the multiple and chaotic reflections and reverberations of sound waves and shocks off the walls of the cavity into which the lion's share of core acoustic energy is being pumped.

There is simultaneous explosion and accretion, enabled by the symmetry breaking. Given symmetry breaking, and without rotation, the explosion naturally generates a cocoon that roughly collimates the outflow. As this cavity is filled with acoustic power, it expands outward, wrapped by the infalling matter being diverted to the "back" side, most of which, during the earlier stages of explosion, is still accreted in sheets/funnels onto the protoneutron star (see Burrows et al. 2006 and Fig. 6). The sound speeds in the exploding cavity are much larger than the initial speed of the outer shock/explosion wave, and the matter is very radiation dominated (as the large entropies in evidence in Fig. 9 would imply). The relatively slow speed (compared with the speeds of the multiple shocks emanating from the core) of the expansion of the blast as it works its way out, deflecting the accreting matter on that one side as it moves, allows the entropy of the cavity to accumulate and grow. Had the cavity expanded on dynamical times, the entropies achieved would have been much lower. In this way, high entropies are achieved.

Since we have yet to follow our simulation explosions to completion, the systematics with progenitor mass of the ejecta entropy, and hence perhaps of the $r$-process yields, is not obvious. Nevertheless, Figure 9 is suggestive.

\section{ANISOTROPIC WIND AND ANISOTROPIC NEUTRINO FLUX}

The explosions we see resemble strong anisotropic winds (see $\S 6)$. A spherical wind imparts no net momentum to the residue; an asymmetric wind imparts a kick and "ablation" force on the accretion streams and core. The recoil implied is a purely hydrodynamical mechanism, whatever the agency of explosion (be it neutrinos or sound), and has two results. First, the recoil due to the anisotropic wind pushes the accretion streams to the opposite side, making the accretion very anisotropic. A fraction of the gravitational energy of accretion is used to continue to excite the inner core $g$-mode oscillation. Because the accretion funnels are supersonic, the coupling to the core is nonlinear. Importantly, the oscillation of the core cannot do work back on the exciting accretion stream(s) that would otherwise damp the core oscillation; any work done is accreted back. Hence, the analogy with the swing that requires a resonance or near-resonance to achieve significant amplitude is not germane. A steady stream onto the core can continue to power the periodic core oscillation, even though there is no intrinsic periodicity to the accretion. The accretion funnel does have a width, which like a rock hitting a pond has associated with it a range of characteristic sizes (read wavelengths/wavenumbers). Due to the dispersion relation of gravity waves between wavenumber and frequency, a whole period spectrum of ripples is generated that contains the period of the $\ell=1$ core $g$-mode (as well as those of many of the higher $\ell$ core $g$-modes).

Second, the recoil provides a kick to the residual core, the proto-neutron star, and this recoil may be the origin of pulsar proper motions. The anisotropic/top-bottom explosion acts like rocket exhaust, and momentum conservation does the rest (A. Burrows et al. 2007, in preparation). The magnitude of the effect can be approximated as follows: the recoil force is equal to $(\sin \alpha) v \dot{M}_{e}$, where $\sin \alpha$ is the average "anisotropy parameter," $v$ is the characteristic wind velocity, and $\dot{M}_{e}$ is the wind mass-loss rate. The "anisotropy parameter" is defined by this expression and is a dimensionless measure of the dipole moment of the momentum density of the ejecta. Its product with the magnitude of the ejecta velocity yields the net specific recoil momentum. For isotropic ejecta, $\sin \alpha$ is zero. The power poured into the supernova "nebula" by the wind is $1 / 2 \dot{M}_{e} v^{2}$. Integrating both of these quantities over time gives the net impulse and explosion energy $(E)$, respectively. The impulse is equal to the residue mass $\left(M_{\mathrm{pn}}\right)$ times the kick velocity $\left(v_{k}\right)$. Taking the ratio of these two expressions results in a formula for the kick velocity:

$$
v_{k}=2 E /\left(M_{\mathrm{pn}} v\right) \sin \alpha .
$$

If we assume that the scale of $v$ is set by a sound speed $\left(\sim 30,000-100,000 \mathrm{~km} \mathrm{~s}^{-1}\right)$, we derive that $v_{k} \sim 1000(E /$ $10^{51}$ ergs) $\sin \alpha \mathrm{km} \mathrm{s}^{-1}$. The average observed/inferred kick speed is 300-400 $\mathrm{km} \mathrm{s}^{-1}$ ( Taylor \& Cordes 1993; Lyne \& Lorimer 1994), so this number is tantalizing. The anisotropy parameter, $\sin \alpha$, can be large but depends on the stochasticity of the flow. This formula works whether the explosion is driven by neutrinos or sound and depends only on the windlike character of the asymmetric explosion and simple momentum conservation. Note that, all else being equal, we would expect larger kicks for larger explosion energies. Whether all else is in fact "equal" remains to be seen, and this correlation may be only statistical. We would also expect that the kicked proto-neutron star and the inner ejecta would move in opposite directions. This is a firm prediction of the model (see also Scheck et al. 2004, 2006). The correlation observed by Wang et al. (2006) between the spin axis and the kick direction would naturally follow in our kick mechanism, as long as the rotation axis sets the axis along which the SASI and the core oscillation break spherical symmetry. This seems plausible, but whether even slight rotation, which otherwise has only modest dynamical effect, can enforce this axis most of the time will require $3 \mathrm{D}$ simulations to determine.

With VULCAN/2D we are also able to ascertain for the first time the magnitude and sign of the impulse due to anisotropic neutrino emissions. We find that during our simulations (to 

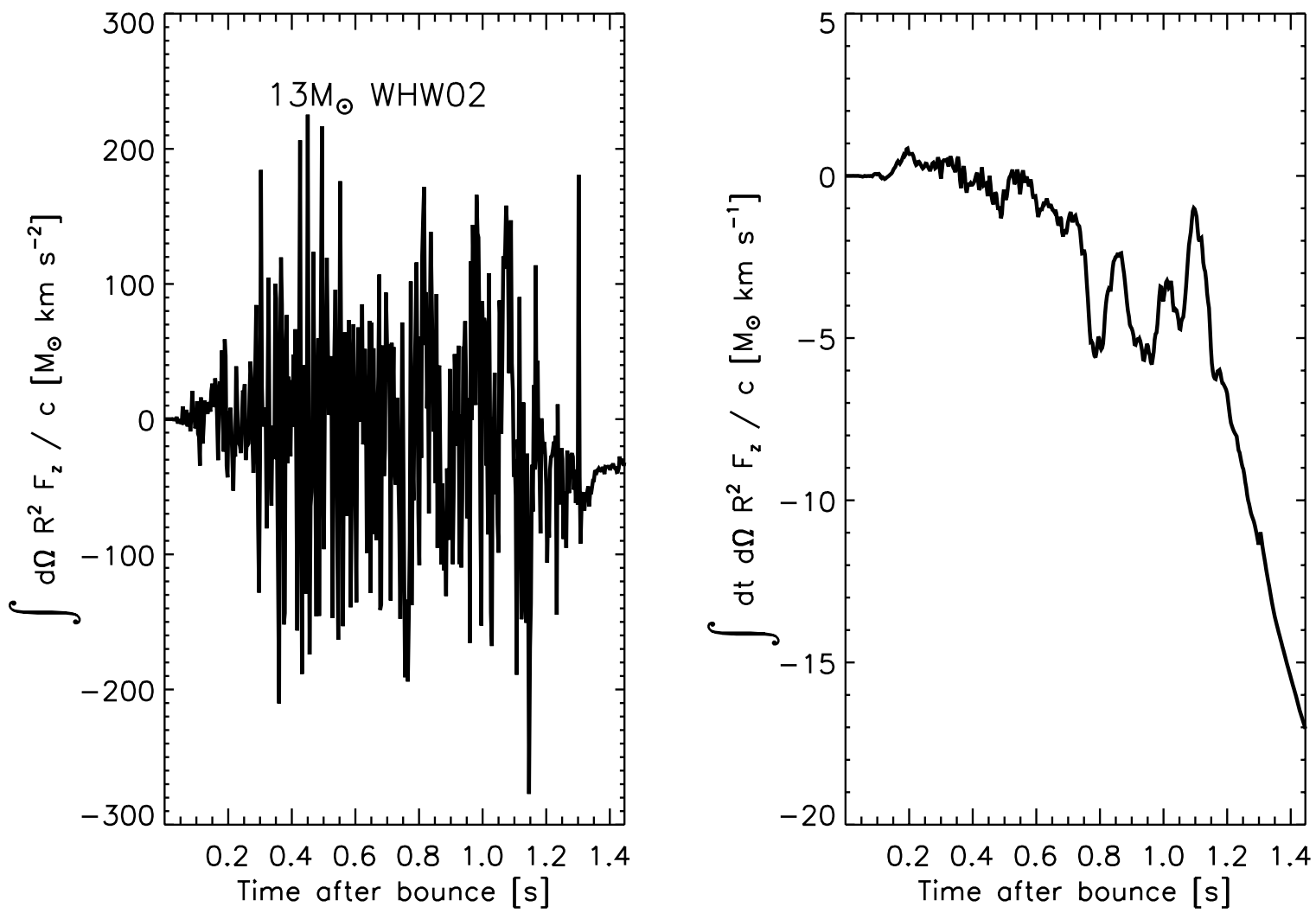

FIG. 10.-Left: Angle-averaged momentum of the emergent neutrinos as a function of the time after bounce for the $13 M_{\odot}$ model of WHW02. This, with a

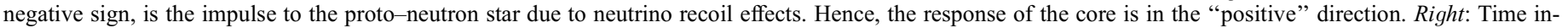
tegral of the instantaneous momentum shown in the left panel as a function of time after bounce. Given the $\sim 1.6 M_{\odot}$ neutron star formed in this collapse after $\sim 1 \mathrm{~s}$, the kick imparted through the anisotropy of the neutrino luminosity is on the order of $\sim 10 \mathrm{~km} \mathrm{~s}^{-1}$ by the end of this calculation but will likely keep accumulating.

approximately $1.5 \mathrm{~s}$ after bounce) the neutrino recoil effect on the core is not large, at most $\sim 50 \mathrm{~km} \mathrm{~s}^{-1}$, but that by the end of our simulations it is still growing and is in the opposite direction to the blast. Hence, after the explosion commences, the impulses on the proto-neutron star due to the matter ejecta and the neutrino radiation $a d d$. Figure 10 depicts both the net force and the accumulated impulse due to neutrinos during our simulation of the postbounce phase of the $13 M_{\odot}$ model of WHW02. The neg- ative sign indicates that the neutrinos are emerging preferentially in the direction of the exploding matter (in this case, "downward;" see Fig. 5), and not toward the accreting side. The small magnitude of the neutrino force during the delay to explosion may seem inconsistent with the very anisotropic accretion. However, the radiation field is much smoother by its nature than the material field. Importantly, the neutrinos are not radiated instantaneously on compression in an accretion column onto the proto-neutron star.
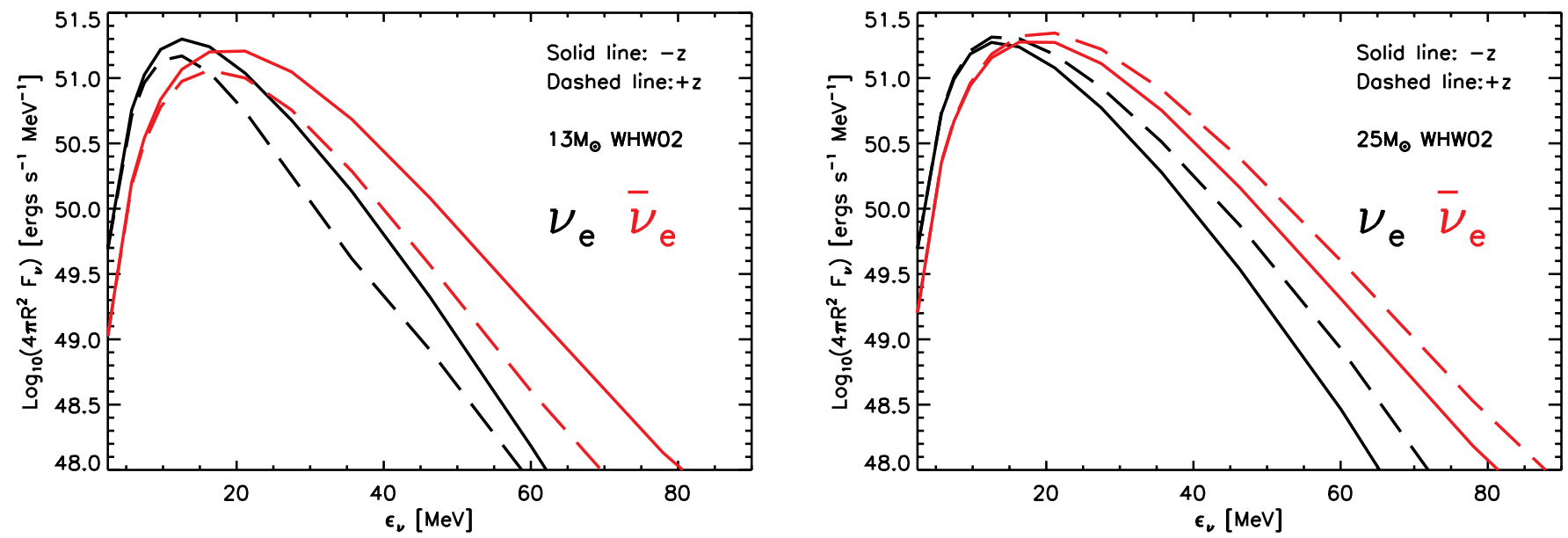

FIG. 11.-Left: Energy spectra of the electron neutrino (black) and antielectron neutrino (red) fluxes along the poles (solid line: negative $z$-direction; dashed line: positive $z$-direction) for the $13 M_{\odot}$ model of WHW02. The fluxes are multiplied by a factor $4 \pi R^{2}$ and are at $1.44 \mathrm{~s}$ after bounce. Right: Same as the left panel, but for the $25 M_{\odot}$ model of WHW02 at $1.42 \mathrm{~s}$ after bounce. Note that the hotter and higher fluxes are in each case in the direction of the explosion, although the two models shown explode in different directions (see Fig. 5). 
The matter is too opaque for immediate reradiation. Rather, the neutrinos emerge after the compressed accreta have spread more uniformly over the inner core and, therefore, are radiated much more isotropically than the matter is accreted. After explosion, the neutrinos can emerge more easily along the direction of the blast, since the material around the neutrinospheres thins out in this direction, and not along the direction experiencing continuing accretion, which, as stated, is more opaque. This result is at odds with the conclusion of Fryer (2004). Figure 11 depicts the spectra of the $\nu_{e}$ and $\bar{\nu}_{e}$ neutrinos in the up and down directions (along the poles) near the end of the simulations of the 13 and $25 M_{\odot}$ models. As Figure 11 demonstrates, the radiation is hotter in the direction of the blasts, which for these models are in opposite directions (see Fig. 5). It is also "brighter" in those directions. This is consistent with the sign of the neutrino recoils shown in Figure 10 and our statement that neutrino impulses and wind recoils add. Hence, we conclude that asymmetric neutrino recoil, integrated even longer than we have in this study, can contribute significantly and naturally to the final pulsar kick. However, we cannot, at this stage, determine whether the matter recoil or the neutrino recoil will eventually prove the more important. Nevertheless, what has emerged from our simulations is a straightforward mechanism for imparting a sizable kick to the residue proto-neutron star that does not require anything but the asymmetric explosion that arises naturally in our calculations without exotic physics.

\section{DISCUSSION AND CONCLUSIONS}

The acoustic and core oscillation mechanism we study in this paper and in Burrows et al. (2006) has a number of features that distinguish it from the classic neutrino mechanism. Here we provide a list of some of its more salient aspects. In arriving at this list, we have been guided by the long-term $(\sim 1.5 \mathrm{~s})$ simulations we have performed for this paper that use the MGFLD version of VULCAN/2D (see the Appendix), liberate the core, and include the full $180^{\circ}$ angular domain. Calculations that do not have the latter two features, that do not include neutrino transfer, or that do not go to very late times cannot be used to study the core oscillation/acoustic component. We find the following:

1. The SASI, when neutrino losses are properly included, does not lead to explosion in and of itself, but creates an anisotropic accretion regime onto the core that eventually leads to nonlinear core pulsation with symmetries ( $\ell$-values) similar to those of the most unstable SASI modes themselves. Both $\ell=1$ and 2 core $g$-mode oscillations can be the most prominent, although the $\ell=1$ mode arises earlier and generally dominates (Burrows et al. 2006).

2. The acoustic power generated by the core oscillation seems to be dumped into the mantle for many seconds after bounce, longer than the standard neutrino mechanism is thought to operate. Furthermore, it can take many hundreds of milliseconds to $\sim 0.6 \mathrm{~s}$ (determined by the progenitor mass accretion rate) before the core oscillation itself achieves large amplitudes.

3. During the early phase of the explosion, the acoustic power steadily punches out through the accreta and generates a collimated explosive flow. This is aided by neutrino heating, which if the acoustic component were suppressed would be the standard underenergetic neutrino-driven wind mechanism (Burrows \& Goshy 1993; Buras et al. 2006a, 2006b). The early net velocities of this flow are low, but before and during this phase a cavity is filled with acoustic energy radiated by the core oscillation. Sound waves bounce off the cavity walls and reverberate in the cavity.

4. At later times, during what would have been the neutrinodriven wind phase in the traditional neutrino-driven explosion, the acoustic mechanism is still aided by neutrino heating at the level of $\sim 10^{50}$ ergs.

5. The inner, early blast is mostly unidirectional and is naturally collimated by the accretion flow that is parted by the blast and diverted to the opposite side of the proto-neutron star. There is simultaneous accretion, explosion, and core oscillation (Burrows et al. 2006).

6. The matter that punches out in the explosion experiences a Kelvin-Helmholtz shear instability that rolls up the interface between the ejecta and the cocooning accreta as the wide-angle "jet" (the early explosion) emerges. However, the coarse outer zoning of our calculations at large radii $(3000-6500 \mathrm{~km})$ is currently insufficient to resolve this interesting phenomenon properly.

7. The explosion is very radiation dominated; most of the explosion energy is initially in internal energy, not kinetic energy. Furthermore, the ejecta have high entropies (100-1000 per baryon per Boltzmann's constant, generated by both neutrino heating and acoustic power), far larger than the generic values (10-50) associated with the early-phase neutrino mechanism. Hence, if the acoustic mechanism works, the early development of the explosion into the star and the associated explosive nucleosynthesis cannot properly be simulated with a piston or a "kinetic energy" bomb.

8. The high entropies suggest that some of the ejecta will undergo $r$-processing (Hoffman et al. 1996; Woosley et al. 1994). This is the first time numerical supernova explosions have simultaneously and naturally generated the conditions that may be necessary for the $r$-process.

9. Our calculations are Newtonian and have been done only with the EOS of Shen et al. (1998). General relativity will change the core oscillation frequencies and so will affect the acoustic power, its evolution, and the timing of the various phases. Consequently, it should eventually be included in the simulations. The incompressibility of nuclear matter will also affect the $g$-mode frequencies; hence, a study of the dependence on the nuclear EOS would be illuminating and may provide diagnostics of the EOS at high densities.

When the SASI is in its vigorous nonlinear phase, the $\ell=1$ oscillations result in quasi-periodic fluctuations in the effective accretion rate and ram pressure on any given side of the inner core. In the canonical neutrino-driven mechanism of supernova explosions, when and after the explosion occurs the pressure around the neutrinospheres decays. When this pressure is sufficiently low, a neutrino-driven wind spontaneously emerges from the inner core, announced and preceded by a secondary shock wave (Burrows \& Goshy 1993; Burrows et al. 1995). This is what happens in the standard neutrino-driven scenario when the flow is semispherical. However, the SASI can set up a situation in which the pressure and ram pressure on one side are such that that side of the core becomes unstable to the emergence of a neutrino-driven wind even before the canonical explosion. In fact, this wind can be the explosion itself and need not be preceded by a primary explosion. This is what Buras et al. (2006b) see for their 11.2 $M_{\odot}$ simulation. However, such an explosion seems generically underenergetic. In the acoustic mechanism, the neutrinos are replaced/dominated by the acoustic power, but the general paradigm in which the SASI leads temporarily/periodically to lower pressures on one side of the core that enable the emergence of an asymmetric wind still obtains. In any case, an aspherical "wind" is a good description of the supernova explosion (see Scheck et al. 2004, 2006; Burrows \& Goshy 1993), and the breaking of spherical symmetry is the key. The latter can also enable simultaneous accretion and explosion, thereby solving the problem of the 
accretion tamp that has bedeviled the theory of the neutrino mechanism for years.

We see in the breaking of spherical symmetry in our simulations and in the unipolar nature of the resulting explosions a natural explanation for the polarizations observed in the inner debris of Type Ic (Wang et al. 2003) and Type II (Leonard et al. 2006) supernovae. Inner ejecta asymmetries of $2: 1$ or $3: 1$ are easily obtained in this model, and in fact in all modern non-MHD explosion models, and do not require MHD jets.

What the actual and relative contributions of sound and neutrinos are to the supernova phenomenon as a function of progenitor remains to be determined and will require even more sophisticated numerical tools than we have applied here to reach a definitive answer. The calculations presented in this paper have several limitations (see the Appendix). We are doing them in 2D; $3 \mathrm{D}$, while out of reach in the short term, will be necessary in the long term. We have employed an MGFLD, not a multiangle, formulation, and the Doppler shift terms in the transport equation have been dropped. While these velocity-dependent terms are very different in the laboratory frame formulation (Hubeny \& Burrows 2006) we have adopted than in the comoving frame formulation of Buras et al. (2006a), they should nevertheless be incorporated. We have used only 16 energy groups; using more $(\gtrsim 20)$ is preferred (Thompson et al. 2003). The spatial resolution in the center is good but can be improved on the outside exterior to $\sim 200 \mathrm{~km}$. The calculations and gravitational field are Newtonian; we expect that general and special relativistic effects can be important on the inside and outside, respectively. The opacities employed are sophisticated, but the neutrino-matter correlation effects at higher densities need a second look.

All in all, the supernova problem has resisted attempts at resolution for too long. In the calculations we present here and in
Burrows et al. (2006), we see new, perhaps provocative, ideas emerge that will require fresh approaches to test and verify them. A potentially new role for the inner core has been highlighted, and the intriguing suggestion that acoustic power might compete with neutrino power to ignite the supernova explosion has been put forward. Furthermore, we find that there is much to explore in the interaction of the core and shock instabilities. Whether these new ingredients in supernova theory are keys or curiosities awaits the next generation of simulations.

We thank Todd Thompson, Rolf Walder, Stan Woosley, John Blondin, and Thierry Foglizzo for fruitful discussions and their insight. We acknowledge support for this work from the Scientific Discovery through Advanced Computing (SciDAC) program of the DOE, grant DE-FC02-01ER41184, and from the NSF under grant AST 05-04947. E. L. thanks the Israel Science Foundation for support under grant $805 / 04$, and C. D. O. thanks the LSU Center for Computation and Technology for providing CPU time on their SuperMike Linux cluster. J. W. M. thanks the Joint Institute for Nuclear Astrophysics (JINA) under NSF grant PHY0216783 for providing a graduate fellowship. This research used resources of the National Energy Research Scientific Computing Center, which is supported by the Office of Science of the US Department of Energy under Contract DE-AC03-76SF00098. We are happy to acknowledge the National Center for Computational Sciences at Oak Ridge for a generous allocation of computer time on Jaguar. Finally, we thank Don Fisher, Youssif Alnashif, and Moath Jarrah for their help generating both color stills and movies associated with this work and Jeff Fookson and Neal Lauver of the Steward Computer Support Group for their invaluable help with the local Beowulf cluster Grendel.

\section{APPENDIX}

\section{VULCAN/2D: A MULTIGROUP, MULTIANGLE RAD/HYDRO CODE AND ITS MGFLD VARIANT}

In this appendix we assemble paragraphs on some of the numerical techniques used in VULCAN/2D, in particular its Multi-Group, Flux-Limited Diffusion realization. Some of this discussion can be found in our other papers using VULCAN/2D (e.g., Livne et al. 2004; Ott et al. 2004; Burrows et al. 2006; Walder et al. 2005; Dessart et al. 2006a, 2006b). We believe that assembling this technical information in one place will better help the reader understand the computational issues that surround such supernova codes in general, and VULCAN/2D in particular. Importantly, although in the past workers focused on improvements in neutrino transfer and transport, the acoustic mechanism requires special attention be paid to the hydrodynamics, grid structure, momentum conservation, gravity solvers, and, we suggest, a moving grid as well, to ensure and maintain good resolution in the inner core. VULCAN/2D is the first supernova code for which these issues have been central considerations.

The code VULCAN/2D uses the explicit hydrodynamic approach described in Livne (1993), with the implicit transport methods discussed in Livne et al. (2004) and Walder et al. (2005). It is a Newtonian, 2D, multigroup, multiangle radiation/hydrodynamics code $^{11}$ with an Arbitrary-Lagrangean-Eulerian (ALE) structure (with remap), a scalar von Neumann-Richtmyer artificial viscosity scheme to handle shocks, and a fast Multi-Group, Flux-Limited Diffusion (MGFLD) variant. The full Boltzmann version discretizes the angular variables using the discrete-ordinates $\left(S_{n}\right)$ method. The code can handle axisymmetric rotation. Velocity terms in the transport sector, such as Doppler shifts, are not included in the code, although advection is. Note that the velocity terms in Eulerian transport are different from the corresponding terms in the comoving frame and that general statements about their relative importance are very frame dependent. We parallelize only in energy groups using MPI, and in 2D no domain decomposition is required. As a result, and in practice, VULCAN/2D is very scalable and the communication overhead is only $2 \%-8 \%$ of the total run time. The fact that domain decomposition (such as is used in FLASH and CACTUS) is not necessary, that we can achieve almost perfect parallelism in energy groups, and that we can include rotation has enabled us to achieve a viable 2D simulation capability.

Note that energy redistribution due to inelastic electron scattering is of only modest import on infall, affecting the trapped electron fraction $\left(Y_{e}\right)$ and entropy $(S)$ by only $\sim 10 \%$. Furthermore, at a neutrino energy of $10 \mathrm{MeV}$, the neutrino-electron scattering cross section is $\sim 100$ times smaller than the dominant cross sections off nucleons. Hence, we have not felt it urgent to include into VULCAN/2D energy redistribution by neutrino-electron scattering. Fortunately, such energy redistribution, because it is subdominant, can be handled semiexplicitly (Thompson et al. 2003), thus avoiding interprocessor communication during an implicit solve. A scheme for this is already written and debugged, since it is used in SESAME (Burrows et al. 2000; Thompson et al. 2003), and is quite

${ }^{11}$ Hence, it is a six-dimensional $[1($ time $)+2($ space $)+2($ angles $)+1($ energy groups $)]$ solver. 
stable. The attempts by others to handle the full energy/angle redistribution problem implicitly have resulted in codes that are thereby slower by many factors (not percent), severely inhibiting their use for explorations in supernova theory.

In 2D, the calculations are axially/azimuthally symmetric, and we use cylindrical coordinates ( $r$ and $z$ ), but the grid points themselves can be placed at arbitrary positions. This allows us to employ a Cartesian grid at the center (typically, the inner $\sim 20-30 \mathrm{~km}$ ) and transition to a spherical grid farther out. The grid resolution is essentially uniform everywhere within this $\sim 20-30 \mathrm{~km}$. A version of this grid structure is plotted in Ott et al. (2004). The Cartesian format in the interior allows us to avoid the severe Courant problems encountered in $2 \mathrm{D}$ by other groups employing grid-based codes due to the inner angular Courant limit, to enable core translational motion, and thereby to perform the calculations in full 2D all the way to the center. In many simulations to date, the inner core has been calculated in 1D and grafted onto an outer region that was handled in 2D (e.g., Burrows et al. 1995; Janka \& Müller 1996; Buras et al. 2003, 2006a, 2006b; Swesty \& Myra 2005, 2006) or has been excised completely (e.g., Blondin et al. 2003; Blondin \& Mezzacappa 2006; Scheck et al. 2004, 2006). The gray SPH simulations of Herant et al. (1994) and Fryer \& Warren $(2002,2004)$ are an exception. Originally, a major motivation for this global 2D feature was the self-consistent investigation of core translational motion and neutron star kicks. However, freeing the core has the advantage that no other multigroup supernova code has of simulating the oscillation of the core and its acoustic radiation.

Note that due to the grid singularity in spherical coordinates at $r=0$ and the inherent difficulties of constructing a reliable finite difference scheme and boundary conditions at that singularity (reflecting?), codes that attempt to include the core in 2D or 3D using spherical coordinates are likely to artificially inhibit translational motion there and, thus, to inhibit $\ell=1 \mathrm{~g}$-modes. Even a simple Galilean transformation/translation of a hydrostatic core, which is what our special grid was designed for, may not be easy when using the standard realizations of a spherical grid in $2 \mathrm{D} / 3 \mathrm{D}$.

Outside the Cartesian mesh, our baseline calculations have typically employed 121-180 angular zones equally spaced over the entire $180^{\circ}$ of the symmetry domain and $\sim 160$ radial shells logarithmically allocated between $\sim 20 \mathrm{~km}$ (generally 10,20 , or $30 \mathrm{~km}$; Dessart et al. 2006a) and the outer radius at $6400-10,000 \mathrm{~km}$. Along the symmetry axis $(r=0)$, we use a reflecting boundary, while at the outer boundary, we use either an outflow or a $v=0$ boundary condition for the matter and a free-streaming boundary condition for the neutrinos.

\section{A1. 2D MULTIGROUP FLUX-LIMITED DIFFUSION OF NEUTRINOS}

The MGFLD implementation of VULCAN/2D is fast and uses a vector version of the flux limiter found in Bruenn (1985; see also Walder et al. 2005). Using the MGFLD variant of VULCAN/2D allows us to perform an extensive study that encompasses the longterm evolution of many models. However, MGFLD is only an approximation to full Boltzmann transport, and differences with the more exact treatment will emerge in the neutrino semitransparent and transparent regimes above the proto-neutron star surface. Nevertheless, inside the neutrinospheres the two-dimensional MGFLD approach provides a very reasonable representation of the multispecies, multigroup neutrino radiation fields.

The evolution of the radiation field is described in the diffusion approximation by a single (group-dependent) equation for the average intensity $J_{g}$ of energy group $g$ with neutrino energy $\varepsilon_{\nu}^{g}$ :

$$
\frac{1}{c} \frac{\partial J_{g}}{\partial t}-\operatorname{div}\left(D_{g} \nabla J_{g}\right)+\sigma_{g}^{a} J_{g}=S_{g}
$$

where the diffusion coefficient is given by $D_{g}=1 / 3 \sigma_{g}$ (and then is flux limited according to the recipe below), the total inverse mean free path ("cross section") is $\sigma_{g}$, and the inverse absorption mean free path (absorption "cross section") is $\sigma_{g}^{a}$. The source term on the right-hand side of equation (A1) is the emission rate of neutrinos of group $g$. Note that equation (A1) neglects inelastic scattering between energy groups.

The finite difference approximation for equation (A1) consists of cell-centered discretization of $J_{g}$. It is important to use cell-centered discretization because the radiation field is strongly coupled to matter and the thermodynamic matter variables are cell centered in the hydrodynamical scheme. The finite difference approximation of equation (A1) is obtained by integrating the equation over a cell. Omitting group indices and cell indices, one gets

$$
V\left[\frac{1}{c \Delta t}\left(J^{n+1}-J^{n}\right)+\sigma^{a} J^{n+1}\right]+\Sigma \boldsymbol{d} \boldsymbol{S}_{i} \cdot \boldsymbol{F}_{i}^{n+1}=V S .
$$

Here $V$ is the volume of the cell and $\boldsymbol{d} \boldsymbol{S}_{i}$ is the face-centered vector "area $\boldsymbol{n}_{i}$, " $\boldsymbol{n}_{i}$ being the outer normal to face $i$. The fluxes $\boldsymbol{F}_{i}$ at internal faces are the face-centered discretization of

$$
\boldsymbol{F}_{i}=-D_{i} \nabla J^{n+1}
$$

where

$$
D_{i}=F L\left(\frac{1}{3 \sigma_{i}}\right)
$$

Our standard flux limiter, following Bruenn (1985) and Walder et al. (2005), is

$$
F L[D]=\frac{D}{1+D|\nabla J| / J}
$$


and approaches free streaming when $D$ exceeds the intensity scale height $J /|\nabla J|$. The fluxes on the outer boundary of the system are defined by free streaming outflow and not by the gradient of $J$. Note that in equation (A3) the fluxes are defined as face quantities, so that they have exactly the same value for the two cells on both sides of that face. The resulting scheme is, therefore, conservative by construction. In order to have a stable scheme in the semitransparent regions (large $D_{g}$ ), we center the variables in equation (A2) implicitly. The fluxes, defined by the intensity at the end of the time step, couple adjacent cells, and the final result is a set of linear equations. The matrix of this system has the standard band structure, and we use direct LU decomposition to solve the linear system. For a moderate grid size the solution of a single linear system of that size does not overload the CPU.

We have parallelized the code according to energy groups. Each processor computes one to a few groups (usually one) and transfers the needed information to the other processors using standard MPI routines. In our baseline runs, we employ 16 energy groups per neutrino species, logarithmically spaced from 1 or $2.5 \mathrm{MeV}$ to 250 or $320 \mathrm{MeV}$. Since we do not split the grid between processors, the parallelization here is very simple. In fact, each processor performs the hydro step on the entire grid. In order to avoid divergent evolution between different processors due to accumulation of machine round-off errors, we copy the grid variables of one chosen processor (processor 0 ) into those of the other processors typically every thousand steps.

\section{A1.1. Coupling Radiation to Matter}

The numerical scheme used in VULCAN consists of a Lagrangean step, followed by a remapping step to the Eulerian grid (Livne 1993). This makes the code similar in this regard to traditional ALE (Arbitrary-Lagrangean-Eulerian) codes. The hydrodynamical variables are all cell centered, except for the position and the velocity, which are node centered. The variables of the radiation field are also cell centered, so that the interaction between the radiation field and matter is properly centered.

The time advancement in both the radiative and the hydrodynamical sectors is computed in the Lagrangean step, whereas the remapping step changes only the spatial discretization of the variables over the numerical grid. We describe here only the Lagrangean scheme. The transport equation itself, for a given source, is always computed in a fully implicit manner.

We first advance the velocity by half a time step:

$$
\boldsymbol{v}^{n+1 / 2}=\boldsymbol{v}^{n}+0.5 \Delta t\left(-\frac{\nabla p^{n}}{\rho^{n}}-\nabla U_{G}\right)
$$

where $p$ is the pressure, $\rho$ is the mass density, $\Delta t$ is the time step, and $U_{G}$ is the gravitational potential (but see $\S$ A3). The position vector is then advanced using

$$
\boldsymbol{r}^{n+1}=\boldsymbol{r}^{n}+\Delta t \boldsymbol{v}^{n+1 / 2}
$$

Denoting by $V$ the volume of a cell, Lagrangean mass conservation takes the form

$$
\rho^{n+1}=\rho^{n} \frac{V^{n}}{V^{n+1}} .
$$

We then solve the adiabatic energy equation for the specific internal energy:

$$
e^{*}=e^{n}-\frac{1}{2}\left(p^{*}+p^{n}\right)\left(\frac{1}{\rho^{n+1}}-\frac{1}{\rho^{n}}\right) .
$$

Equation (A9) is iterated to convergence. At this stage, we compute new cross sections and emission sources

$$
S_{\mathrm{em}}=\sum_{g} \sigma_{g}^{a} J_{g}^{\mathrm{eq}}
$$

where $J_{g}^{\text {eq }}$ is the LTE intensity (a function of density, temperature, and composition). Using those cross sections and sources, we solve the transport equation. The net change in the radiation energy density is given by

$$
\Delta E_{r}=\Delta t \sum_{g} \sigma_{g}^{a}\left(J_{g}^{\mathrm{eq}}-J_{g}^{n+1}\right)
$$

and this is also minus the net change in the matter energy density. Consequently, we compute the final energy density, pressure, and temperature using

$$
e^{n+1}=e^{*}-\Delta E_{r} / \rho^{n+1}
$$

and the EOS.

For the supernova problem, we also need to compute the degree of neutronization of matter due to electron capture and other charged-current processes (Burrows \& Thompson 2004). We obtain

$$
Y_{e}^{n+1}=Y_{e}^{n}-\Delta t\left[\frac{\sum_{g} \sigma_{g}^{a}\left(J_{g}^{\mathrm{eq}}-J_{g}^{n+1}\right)}{\varepsilon_{\nu}^{g}}\right] \frac{1}{N_{a} \rho},
$$




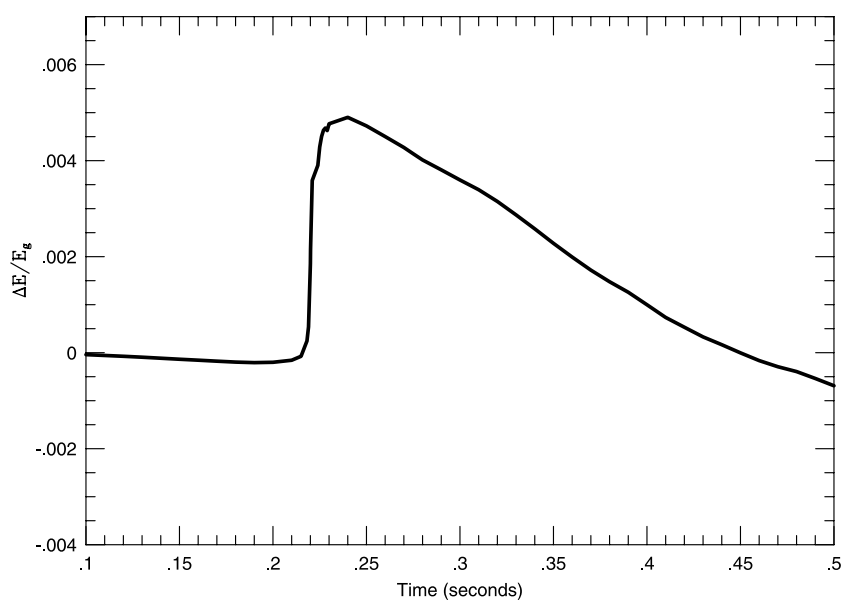

Fig. 12.-Plot of $\Delta E / E_{g}$ vs. time (from the start of the calculation), where $\Delta E$ is the total energy conservation error and $E_{g}$ is the gravitational potential energy. This figure is for the simulation performed by Ott et al. (2006a) of the $11 M_{\odot}$ progenitor model of WW95 that was rotated to have a rapid initial spin of 2.68 rad s ${ }^{-1}$ in the core. For rotating models, energy conservation will generally be worse than for nonrotating models. The dimensionless ratio $\Delta E / E_{g}$ is a useful measure of the degree to which energy is conserved during a simulation using VULCAN/2D. The bump on the plot at $\sim 0.22 \mathrm{~s}$ occurs at bounce. See $\S \mathrm{A} 3$ for a discussion.

where $N_{a}$ is Avogadro's number and $Y_{e}$ is the electron fraction. Finally, we advance the velocity due to the new matter pressure by a further half time step and due to the radiation pressure $\left(\boldsymbol{F}_{\mathrm{rad}}^{\text {node }}\right)$ by a full time step:

$$
\boldsymbol{v}^{n+1}=\boldsymbol{v}^{n+1 / 2}+0.5 \Delta t\left[\frac{1}{\rho^{n+1}}\left(-\nabla p^{n+1}+2 \boldsymbol{F}_{\text {rad }}^{\text {node }}\right)-\nabla U_{G}\right] .
$$

The radiation force at grid nodes is evaluated by a simple averaging process using the radiation force at cell centers and the definition of the radiation flux (eq. [A3]).

\section{A2. ADVECTION OF ANGULAR MOMENTUM}

Since VULCAN/2D uses an Eulerian grid, when we study rotating models the specific angular momentum is advected with the flow in the same manner as linear momentum components. In so doing, we maintain global angular momentum conservation by construction. Note that the axis in cylindrical coordinates is a singularity and, as such, is prone to slightly larger errors than can be expected elsewhere on the grid. However, the actual volume of the cells nearest the axis is very small and the errors do not affect the overall flow. In the past (Walder et al. 2005) we have estimated such departures near the singularity for rotating models to be no more than $\sim 10 \%$ in any flow variable and to be much smaller elsewhere.

\section{A3. GRAVITY AND POISSON SOLVERS}

Gravity is a key force in multidimensional astrophysical hydrodynamics. However, many calculations in the past have employed only the monopole term and/or have complemented the gravitational force term $\left(F_{G}\right)$, written as a gradient of a potential in the momentum equation (eq. [A6]) with a corresponding $\boldsymbol{v} \cdot \boldsymbol{F}_{G}$ term in the energy equation. The latter approach is perfectly reasonable but, given the inherently approximate nature of finite difference realizations of the partial differential equations, does not guarantee momentum conservation, nor consistency between the momentum and energy equations when written in Eulerian form. To address this, we have implemented a version of the code in which the $z$-component of gravity appears as the divergence of a stress tensor (Shu 1992, p. 46; Xulu 2003). This ensures, in principle, the conservation of momentum in that direction, or at least guarantees that in fact the gravitational force of mass parcel " $\mathrm{A}$ " on "B" is equal and opposite to the gravitational force of mass parcel "B" on "A."

Currently, there are in VULCAN/2D two Poisson solvers: a multipole solver and a grid solver. The multipole solver is a standard Legendre expansion, and we typically employ 20-33 terms. For the potential calculations, one generally needs a special auxiliary grid, which is not identical with that of our complex hydro grid. This leads to a number of interpolations between the grids, which can introduce significant numerical errors. Most importantly, with the multipole solver conservation of total energy is poor through bounce and later. Conservation of total energy is much better with the other solver, the grid solver, and the numerical noise in the core region is significantly reduced when we employ it. However, due to the unavoidable operator split between the hydro and the gravity calculations, it is generically hard to get good total energy conservation. VULCAN/2D generally conserves energy to an average of better than $0.4 \%$ in terms of $\Delta E / E_{\text {grav }}$, with the worst energy conservation phase near core bounce. Figure 12 depicts $\Delta E / E_{\text {grav }}$ versus time for the published $11.0 M_{\odot}$ run from Ott et al. (2006a) that includes neutrinos and has a rapid initial spin of $2.68 \mathrm{rad} \mathrm{s}^{-1}$ in the core. Rotation generally increases the error. The major reasons $\Delta E / E_{\text {grav }}$ is not zero are as follows: (1) The code is not automatically conservative and, hence, the gravitational term in the energy equation the code "thinks" it uses, given the finite difference approach, is different from our postprocessed calculation of $\int \frac{1}{2} \rho \Phi d V$. Differences of a percent in this can cause large differences that may or may not be meaningful. (2) A similar point can be made concerning the neutrino energy integration for logarithmically distributed energy gridding: how accurately can one integrate under a curve that is sparce at higher energies? (3) The 2D ALE code uses a remap step that is not "perfect" when the velocities change fast near bounce. (4) A predictor/corrector step, which we do not have, would give us higher order accuracy in time. (5) There are slight differences in the 
finite difference treatment of the matter and radiation source terms, which are formally equal and opposite. For a comparison, Liebendörfer et al. (2004) quote errors of $\sim 0.005$ for the same quantity at the end of their 1D calculations, similar to the peak problem we show in Figure 12.

The grid solver, which we employ in this paper, uses the standard finite element method (FEM), which is adequate for unstructured grids, to get the potential at grid nodes. In axial symmetry Poisson's equation takes the form

$$
\Delta \Phi=\frac{1}{r} \frac{\partial}{\partial r}\left(r \frac{\partial \Phi}{\partial r}\right)+\frac{\partial^{2} \Phi}{\partial z^{2}}=-4 \pi G \rho .
$$

Let $\left\{\alpha_{i}(r, z)\right\}$ be a set of interpolation functions that span our FEM approximation. Multiplying equation (A15) by $\alpha_{i}$ and integrating by parts over the entire domain, one gets

$$
-\iint \nabla \Phi \nabla \alpha_{i} r d r d z+\{\text { surface }- \text { integral }\}=-4 \pi G \iint \rho \alpha_{i} r d r d z .
$$

In particular, if we expand $\Phi$ using the set $\left\{\alpha_{i}\right\}$, specifically

$$
\Phi(r, z)=\Sigma_{j} \Phi_{j} \alpha_{j}(r, z),
$$

where $\Phi_{j}$ is the value of $\Phi$ at node $j$, we get a linear system of the form

$$
A \Phi=\boldsymbol{B},
$$

where

$$
A_{i j}=-\iint \nabla \alpha_{i} \nabla \alpha_{j} r d r d z
$$

and

$$
B_{i}=-4 \pi G \iint \rho \alpha_{i} r d r d z
$$

The matrix $A$ has good qualities if we choose $\left\{\alpha_{i}\right\}$ to be continuous, positive, and local, with the following specifications: $\alpha_{i}\left(r_{j}, z_{j}\right)=\delta_{i j}$ at the nodes of the grid, $\alpha_{i}=0$ in any zone not containing node $i$, and $\Sigma_{i} \alpha_{i}(r, z)=1$ everywhere. In practice, we employ bilinear interpolation functions in each zone. The integrals given by equation (A19) are computed once, using numerical integration, and the integrals given by equation (A20) are computed each time step.

Note, however, that the grid solver needs boundary values, and for this we take the zeroth moment $\Phi_{b}=-G M / R_{b}$, where $R_{b}$ here is the distance between a boundary point and the center of mass (which is usually very close to the center of the grid). This approximation is good for large outer radii where the potential drops by orders of magnitude compared with that at the center.

\section{A3.1. Gravity and the Conservation of Linear Momentum}

Importantly, we incorporate the gravitational force along the symmetry axis in an automatically momentum-conserving fashion by writing it in divergence form.

Gravity enters the momentum equation

$$
\frac{\partial(\rho \boldsymbol{v})}{\partial t}=-\nabla P+\rho \nabla \Phi
$$

via the potential $\Phi$ defined by the Poisson equation

$$
\Delta \Phi=-4 \pi G \rho .
$$

In equation (A6), the gravity term is not in conservative form and, therefore, it is not useful for obtaining a conservative numerical scheme. To overcome this, we use equation (A22) to obtain

$$
\boldsymbol{f}_{g}=\rho \nabla \Phi=-\frac{1}{4 \pi G} \Delta \Phi \nabla \Phi .
$$

In Cartesian coordinates, the right-hand side of equation (A23) can be written in a divergence form:

$$
\boldsymbol{f}_{g}=\operatorname{div}(\mathbf{S})
$$

where the gravitational stress tensor $\mathbf{S}$ is derived from $\boldsymbol{g}=\nabla \Phi$ (Shu 1992, p. 47):

$$
\mathbf{S}_{i j}=-\frac{1}{4 \pi G}\left(g_{i} g_{j}-\frac{1}{2}|\boldsymbol{g}|^{2} \delta_{i j}\right) .
$$


In cylindrical coordinates $(r, z)$ with axial symmetry we use the same idea with the Laplacian $\Delta \Phi=(1 / r)\left[\partial\left(r g_{r}\right) / \partial r\right]+\left[\partial\left(g_{z}\right) / \partial z\right]$. Direct calculation yields

$$
f_{z}=-\frac{1}{4 \pi G}\left[\frac{1}{r} \frac{\partial\left(r g_{r} g_{z}\right)}{\partial r}+\frac{1}{2} \frac{\partial\left(g_{z}^{2}-g_{r}^{2}\right)}{\partial z}\right]
$$

and

$$
f_{r}=-\frac{1}{4 \pi G}\left\{\frac{1}{2 r} \frac{\partial\left[r\left(g_{r}^{2}-g_{z}^{2}\right)\right]}{\partial r}+\frac{\partial\left(g_{r} g_{z}\right)}{\partial z}+\frac{1}{2 r}\left(g_{r}^{2}+g_{z}^{2}\right)\right\} .
$$

Note that the $z$-component of the momentum equation (eq. [A6]) now has a divergence form and, therefore, can be integrated to give a conservative finite difference scheme.

In practice, we compute the potential on grid nodes and then compute $g$ at cell centers. The forces are computed by integrating equations (A24) and (A25) over a control volume around a node, where the boundary line of this control volume passes through the centers of the cells circling that node. The conservative form of $f_{z}$ expresses itself in the scheme by having contributions from boundary terms only.

\section{A4. GRID MOTION}

In full $180^{\circ}$ simulations the core has the freedom to escape the center of the grid, where the resolution is finest. Experience shows that in very long simulations the motion of the core off the center of the grid is numerically unstable and can lead to an artificial "kick." In order to avoid this situation, VULCAN/2D has an option to move the grid after bounce to maintain the best zoning under the core, whether it moves or not, while at the same time tracking this core motion. This feature ensures that the highest resolution is placed under most of the mass. Adding a constant $\delta v_{z}$ to $v_{z}$ everywhere does not change anything in the dynamics. Generally, we calculate the position of the center of mass of the inner material above a density of $10^{12} \mathrm{~g} \mathrm{~cm}^{-3}$ and execute the grid motion every time step to position the grid center at this point. As a test, we have allowed grid motion every 100 time steps, and the results fall right on top of those done every time step. This procedure typically ensures that the center of mass stays at the center of the grid to within $\sim 10-100 \mathrm{~m}$.

\section{A5. SEEDS FOR INSTABILITIES}

The instabilities that develop in the early stages of the postbounce phase are seeded by the slight perturbations introduced due to the nonorthogonal shape of the grid regions that affect the transition from the inner Cartesian grid to the outer spherical grid (see Fig. 4 in Ott et al. 2004) and by noise at the 1 part in $\sim 10^{6}$ level in the EOS table interpolation. Since the resulting turbules execute more than 20 overturns during the initial phase of convective instability, and this convective phase reaches a quasi-steady state, the initial conditions and the initial perturbations are completely lost in subsequent evolution. The seeds for the later shock instability are the nonlinear convective structures that arise in the first postbounce tens of milliseconds. Beyond these, we introduce no artificial numerical perturbations.

\section{A6. MICROPHYSICS}

We employ the EOS of Shen et al. (1998), since it correctly incorporates alpha particles and is more easily extended to lower densities and higher entropies than the standard Lattimer \& Swesty (1991) EOS. The neutrino-matter interaction physics is taken from Thompson et al. (2003) and Burrows \& Thompson (2004). The tables generated in $T / \rho / Y_{e} /$ neutrino species space incorporate all relevant scattering, absorption, and emission processes. We follow separately the electron neutrino $\left(\nu_{e}\right)$ and antielectron neutrino $\left(\bar{\nu}_{e}\right)$, but for computational efficiency we lump the four remaining known neutrinos into " $\nu_{\mu}$ " bins in the standard fashion. Our baseline models have 16 energy groups for each species, distributed logarithmically from 1 or $2.5 \mathrm{MeV}$ to 250 or $320 \mathrm{MeV}$. As implied above, neutrino radiation pressure is handled consistently with a local " $-\kappa F_{\nu} / c$ " body force term in the momentum equation. Due to extreme matter suppression effects, we have not felt it necessary to incorporate the effects of neutrino oscillations but have developed a fully consistent formalism to do so (Strack \& Burrows 2005).

Akiyama, S., Wheeler, J. C., Meier, D. L., \& Lichtenstadt, I. 2003, ApJ, 584, 954

Bethe, H., \& Wilson, J. R. 1985, ApJ, 295, 14

Blondin, J. M., \& Mezzacappa, A. 2006, ApJ, 642, 401

Blondin, J. M., Mezzacappa, A., \& DeMarino, C. 2003, ApJ, 584, 971

Bruenn, S. W. 1985, ApJS, 58, 771

Buras, R., Janka, H.-Th., Rampp, M., \& Kifonidis, K. 2006a, A\&A, 457, 281

Buras, R., Rampp, M., Janka, H.-Th., \& Kifonidis, K. 2003, Phys. Rev. Lett., 90, 241101

. 2006b, A\&A, 447, 1049

Burrows, A. 1987, ApJ, 318, L57

Burrows, A., \& Goshy, J. 1993, ApJ, 416, L75

Burrows, A., Hayes, J., \& Fryxell, B. A. 1995, ApJ, 450, 830

Burrows, A., \& Lattimer, J. M. 1986, ApJ, 307, 178

\section{REFERENCES}

Burrows, A., Livne, E., Dessart, L., Ott, C. D., \& Murphy, J. 2006, ApJ, 640, 878 Burrows, A., \& Thompson, T. A. 2004, in Core Collapse of Massive Stars, ed. C. Fryer (Dordrecht: Kluwer), 133

Burrows, A., Young, T., Pinto, P., Eastman, R., \& Thompson, T. 2000, ApJ, 539,865

Dessart, L., Burrows, A., Livne, E., \& Ott, C. D. 2006a, ApJ, 645, 534

Dessart, L., Burrows, A., Ott, C. D., Livne, E., Yoon, S.-Y., \& Langer, N. 2006b, ApJ, 644, 1063

Ferrari, V., Miniutti, G., \& Pons, J. A. 2003, MNRAS, 342, 629

Foglizzo, T. 2001, A\&A, 368, 311

. 2002, A\&A, 392, 353

Foglizzo, T., Galletti, P., \& Ruffert, M. 2005, A\&A, 435, 397

Foglizzo, T., Galletti, P., Scheck, L., \& Janka, H.-Th. 2007, ApJ, 654, 1006

Foglizzo, T., Scheck, L., \& Janka, H.-Th. 2006, ApJ, 652, 1436 
Foglizzo, T., \& Tagger, M. 2000, A\&A, 363, 174

Fröhlich, C., Hauser, P., Liebendörfer, M., Martinez-Pinedo, G., Bravo, E., Hix, W. R., Zinner, N. T., \& Thielemann, F.-K. 2005, Nucl. Phys. A, 758, 27

Fröhlich, C., Martinez-Pinedo, G., Liebendörfer, M., Thielemann, F.-K., Bravo, E., Hix, W. R., Langanke, K., \& Zinner, N. T. 2006a, Phys. Rev. Lett., 96, 142502

Fröhlich, C., et al. 2006b, ApJ, 637, 415

Fryer, C. L. 2004, ApJ, 601, L175

Fryer, C. L., \& Warren, M. 2002, ApJ, 574, L65 2004, ApJ, 601, 391

Hamuy, M. 2003, ApJ, 582, 905

Heger, A., Woosley, S. E., \& Spruit, H. 2005, ApJ, 626, 350

Herant, M., Benz, W., Hix, W. R., Fryer, C. L., \& Colgate, S. A. 1994, ApJ, 435,339

Hoffman, R. D., Woosley, S. E., Fuller, G. M., \& Meyer, B. S. 1996, ApJ, 460, 478

Hubeny, I., \& Burrows, A. 2006, ApJ, submitted (astro-ph/0609049)

Janka, H.-Th., Buras, R., Kifonidis, K., Marek, A., \& Rampp, M. 2005a, in IAU Colloq. 192, Cosmic Explosions, On the 10th Anniversary of SN 1993J, ed. J. M. Marcaide \& K. W. Weiler (Berlin: Springer), 253

Janka, H.-Th., Buras, R., Kitaura Joyanes, F. S., Marek, A., Rampp, M., \& Scheck, L. 2005b, Nucl. Phys. A, 758, 19

Janka, H.-Th., \& Müller, E. 1996, A\&A, 306, 167

Kitaura, F. S., Janka, H.-Th., \& Hillebrandt, W. 2006, A\&A, 450, 345

Kotake, K., Sato, K., \& Takahashi, K. 2006, Rep. Prog. Phys., 69, 971

Lattimer, J. M., \& Swesty, F. D. 1991, Nucl. Phys. A, 535, 331

Leonard, D. C., et al. 2006, Nature, 440, 505

Liebendörfer, M., Messer, O. E. B., Mezzacappa, A., Bruenn, S. W., Cardall, C. Y., \& Thielemann, F.-K. 2004, ApJS, 150, 263

Liebendörfer, M., Mezzacappa, A., Thielemann, F.-K., Messer, O. E. B., Hix, W. R., \& Bruenn, S. W. 2001, Phys. Rev. D, 63, 103004

Livne, E. 1993, ApJ, 412, 634

Livne, E., Burrows, A., Walder, R., Thompson, T. A., \& Lichtenstadt, I. 2004, ApJ, 609, 277

Lyne, A., \& Lorimer, D. R. 1994, Nature, 369, 127

Mayle, R., \& Wilson, J. R. 1988, ApJ, 334, 909

Miralles, J. A., Pons, J. A., \& Urpin, V. 2004, A\&A, 420, 245

Moiseenko, S. G., Bisnovatyi-Kogan, G. S., \& Ardeljan, N. V. 2006, MNRAS, 370,501
Nomoto, K., \& Hashimoto, M. 1988, Phys. Rep., 163, 13

Obergaulinger, M., Aloy, M. A., \& Müller, E. 2006, A\&A, 450, 1107

Ott, C. D., Burrows, A., Dessart, L., \& Livne, E. 2006a, ApJS, 164, 130 . 2006b, Phys. Rev. Lett., 96, 201102

Ott, C. D., Burrows, A., Livne, E., \& Walder, R. 2004, ApJ, 600, 834

Pruet, J., Hoffman, R. D., Woosley, S. E., Janka, H.-Th., \& Buras, R. 2006, ApJ, 644, 1028

Pruet, J., Woosley, S. E., Buras, R., Janka, H.-Th., \& Hoffman, R. D. 2005, ApJ, 623, 325

Scheck, L., Kifonidis, K., Janka, H.-Th., \& Müller, E. 2006, A\&A, 457, 963

Scheck, L., Plewa, T., Janka, H.-Th., Kifonidis, K., \& Müller, E. 2004, Phys. Rev. Lett., 92, 011103

Shen, H., Toki, H., Oyamatsu, K., \& Sumiyoshi, K. 1998, in Proceedings of the International Conference on Neutron Stars and Pulsars, ed. N. Shibazaki et al. (Tokyo: Universal Academy Press), 157

Shu, F. H. 1992, The Physics of Astrophysics, Vol. II (Mill Valley: University Science Books)

Strack, P., \& Burrows, A. 2005, Phys. Rev. D, 71, 093004

Swesty, F. D., \& Myra, E. S. 2005, in Open Issues in Core Collapse Supernova Theory, ed. A. Mezzacappa \& G. M. Fuller (London: World Scientific), 176 2006, J. Phys. Conf. Ser., 16, 380

Taylor, J. H., \& Cordes, J. M. 1993, ApJ, 411, 674

Thompson, T. A., Burrows, A., \& Meyer, B. S. 2001, ApJ, 562, 887

Thompson, T. A., Burrows, A., \& Pinto, P. A. 2003, ApJ, 592, 434

Thompson, T. A., Quataert, E., \& Burrows, A. 2005, ApJ, 620, 861

Walder, R., Burrows, A., Ott, C. D., Livne, E., Lichtenstadt, I., \& Jarrah, M. 2005, ApJ, 626, 317

Wang, C., Lai, D., \& Han, J. L. 2006, ApJ, 639, 1007

Wang, L., Baade, D., Höflich, P., \& Wheeler, J. C. 2003, ApJ, 592, 457

Wilson, J. R., \& Mayle, R. 1988, Phys. Rep., 163, 63 . 1993, Phys. Rep., 227, 97

Woosley, S. E., Heger, A., \& Weaver, T. A. 2002, Rev. Mod. Phys., 74, 1015 (WHW02)

Woosley, S. E., \& Hoffman, R. D. 1992, ApJ, 395, 202

Woosley, S. E., \& Weaver, T. A. 1995, ApJS, 101, 181 (WW95)

Woosley, S. E., Wilson, J. R., Mathews, G. J., Hoffman, R. D., \& Meyer, B. S. 1994, ApJ, 433, 229

Xulu, S. S. 2003, preprint (hep-th/0308070) 